\title{
Production and attenuation of cosmic-ray boosted dark matter
}

\author{
Chen $\mathrm{Xia}^{a}$, Yan-Hao $\mathrm{Xu}^{a}$, and Yu-Feng Zhou ${ }^{a, b, c}$ \\ ${ }^{a}$ CAS key laboratory of theoretical Physics, Institute of Theoretical Physics, \\ Chinese Academy of Sciences, Beijing 100190, China; \\ School of Physics, University of Chinese Academy of Sciences, Beijing 100049, China; \\ ${ }^{b}$ School of Fundamental Physics and Mathematical Sciences, \\ Hangzhou Institute for Advanced Study, UCAS, Hangzhou 310024, China; \\ ${ }^{c}$ International Centre for Theoretical Physics Asia-Pacific, Beijing/Hangzhou, China.
}

(Dated: February 8, 2022)

\begin{abstract}
Light sub-GeV halo dark matter (DM) particles up-scattered by high-energy cosmic-rays (CRs) (referred to as CRDM) can be energetic and become detectable by conventional DM direct detection experiments. We perform a refined analysis on the exclusion bounds of the spin-independent DMnucleon scattering cross section $\sigma_{\chi p}$ in this approach. For the exclusion lower bounds, we determine the parameter of the effective distance $D_{\text {eff }}$ for CRDM production using spatial-dependent CR fluxes and including the contributions from the major heavy $\mathrm{CR}$ nuclear species. We obtain $D_{\text {eff }} \simeq 9 \mathrm{kpc}$ for CRDM particles with kinetic energy above $\sim 1 \mathrm{GeV}$, which pushes the corresponding exclusion lower bounds down to $\sigma_{\chi p} \sim 4 \times 10^{-32} \mathrm{~cm}^{2}$ for DM particle mass at $\mathrm{MeV}$ scale and below. For the exclusion upper bounds from Earth attenuation, previous estimations neglecting the nuclear form factor leaded to typical exclusion upper bounds of $\sigma_{\chi p} \sim \mathcal{O}\left(10^{-28}\right) \mathrm{cm}^{2}$ from the XENON1T data. Using both the analytic and numerical approaches, we show that for CRDM particles, the presence of the nuclear form factor strongly suppresses the effect of Earth attenuation. Consequently, the cross section that can be excluded by the XENON1T data can be a few orders of magnitude higher, which closes the gap in the cross sections excluded by the XENON1T experiment and that by the astrophysical measurements such that for the cosmic microwave background (CMB), galactic gas cloud cooling, and structure formation, etc..
\end{abstract}




\section{INTRODUCTION}

Although compelling astrophysical evidence supports the existence of dark matter (DM) in the Universe, whether or not DM participates non-gravitational interactions remains unknown. Underground DM direct detection (DD) experiments search for recoil signals from the possible scatterings between the halo DM particles and target nuclei. Stringent constraints on the DM-nucleon scattering cross section have been established, which reaches $\sigma_{\chi p} \leq \mathcal{O}\left(10^{-46}\right) \mathrm{cm}^{2}$ for DM particle mass around $\mathcal{O}(10) \mathrm{GeV}[1,2]$. As the typical detection threshold of the current experiments is of $\mathcal{O}(\mathrm{keV})$, for the standard halo DM with a typical escape velocity of $\sim 540 \mathrm{~km} \mathrm{~s}^{-1}$, the current DD experiments lose sensitivity rapidly to DM particles with mass below a few $\mathrm{GeV}$. The reason is that for lighter halo DM particles the kinetic energy is lower, and the energy transfer to the target nuclei is less efficient. Several physical processes have been considered to lower the detection threshold such as using additional photon emission in the inelastic scattering process [3] and the Migdal effect [4, 5], etc..

The same DM-nucleus scattering process can leave imprints in some astrophysical observables, which can be used to place constraints on the scattering cross section. The resulting constraints can be applied to much lower DM particle masses unreachable to the current DM direct detection experiments, but the constraints are in general much weaker. For instance, from the spectral distortion of the cosmic microwave background (CMB), a constraint of $\sigma_{\chi p} \lesssim 5 \times 10^{-27} \mathrm{~cm}^{2}$ for $m_{\chi}$ in the range of $1 \mathrm{keV}-\mathrm{TeV}$ can be obtained [6]. The measurement of the gas cooling rate of the Leo $\mathrm{T}$ dwarf galaxy can also lead to a constraint of $\sigma_{\chi p} \lesssim 3 \times 10^{-25} \mathrm{~cm}^{2}$ for $m_{\chi} \lesssim 1 \mathrm{MeV}[7,8]$. The constraints from the population of Milky Way (MW) satellite galaxies can reach $\sigma_{\chi p} \lesssim 6 \times 10^{-30} \mathrm{~cm}^{2}$ for $m_{\chi} \lesssim 10 \mathrm{keV}$ [9]. Other astrophysical constraints from exoplanet [10] and cosmic-ray (CR) down scattering [11], etc. have also be considered.

Recently, it was shown that more stringent constraints can be derived from the elastic scatterings between cosmic-ray (CR) particles and DM particles. High-energy CRs in the Galaxy can scatter off halo DM particles, which results in the energy-loss of CRs [11], the production of $\gamma$-rays $[12,13]$, and energy-boost of DM particles [14-16], etc.. In the last process, a small but irreducible component of DM (referred to as CRDM) can obtain very high kinetic energies. They can scatter again off the target nuclei in the detectors of the underground DM direct detection experiments, and deposit sufficient energy to cross the detection threshold, which greatly extends the sensitivity of the current experiments to subGeV DM particles [14-24]. In this approach the constraints on a constant DM-nucleon (DM-electron) spin-independent scattering cross section can reach $\sim 10^{-31}\left(10^{-34}\right) \mathrm{cm}^{2}$ for DM particle mass down to at least $\sim 0.1 \mathrm{MeV}(\sim 1 \mathrm{eV})[14,15]$ (for constraints on energydependent and inelastic scattering cross sections, see e.g. [20-22, 25-28]). An advantage of this approach is that the obtained constraints are insensitive to the DM particle mass. This is related to the spectra feature of the CR flux. In Ref. [24], it was shown that if the CR flux follows a power law of $E^{-\alpha}$ with $\alpha=3$, the DM particle mass dependence in the CRDM flux canceled out completely, and consequently the derived constraints are DM particle mass independent. Since the observed CR flux is indeed close to the $\alpha=3$ case, especially for 
CR energy above PeV, the constraints are highly DM particle mass independent and can be extrapolated to very low DM particles mass far below eV scale. These constraints only depend on DM-nucleon scattering in the present-day local Universe, which is complementary to those derived using the observables of the earlier epochs of the Universe, such as that from BBN [29], CMB [6, 30-32], Lyman- $\alpha$ forest [33], and $21 \mathrm{~cm}$ radiations [30], etc..

For large cross sections above $\mathcal{O}\left(10^{-30}\right) \mathrm{cm}^{2}$, the DM energy loss due to the same DM elastic scattering off the nuclei within the crust of the Earth becomes significant. For large enough cross sections, the DM particle can lose most of its kinetic energy before reaching the detector, which leads to an exclusion upper bound ("blind spots") on the scattering cross section for underground DM detection experiments. For the leading experiment of XENON1T with depth around $1.4 \mathrm{~km}$, the typical exclusion upper bound is around $\mathcal{O}\left(10^{-28}\right) \mathrm{cm}^{2}$ when the effect of the nuclear form factor is neglected [14, 23-25]. Thus there exists a gap in the cross section which can be excluded by the underground DM direct detection experiments and that by the astrophysical observables. Additional experimental data have to be considered to close the gap such as using other underground experiments with shallower sites [16].

In this work, we perform a refined analysis on the exclusion bounds on DM-nucleon scattering cross section $\sigma_{\chi p}$ in this approach using CR-boosted DM (CRDM). For the exclusion lower bounds, making use of the numerical code GalProp for CR propagation in the Galaxy, we for the first time consider the inhomogeneity of the CR distribution and determine an important parameter of effective distance $D_{\text {eff }}$ which is often considered as an free phenomenological parameter in the literature. Using spatial-dependent CR fluxes and including the contributions from the major $\mathrm{CR}$ nuclear species from $\mathrm{H}$ to $\mathrm{Ni}$, we obtain $D_{\text {eff }} \simeq 9 \mathrm{kpc}$ for CRDM particle with kinetic energy above $\sim 1 \mathrm{GeV}$. Including CR species heavier than helium leads to a factor of two enhancement of the total CRDM flux. The corresponding exclusion lower bounds can reach $\sigma_{\chi p} \sim 4 \times 10^{-32} \mathrm{~cm}^{2}$ for DM particles with mass at $\mathrm{MeV}$ scale and below. For the detection upper bounds, we numerically simulate the effect of Earth attenuation of CRDM with the effect of nuclear form factor fully taken into account. The numerical simulation of Earth attenuation is based on the DarkProp code which is publicly available at [34]. We find that for relativistic CRDM particles, the nuclear form factor significantly reduce the stopping-power of the Earth's crust. After consistently including the form factors in the simulation, the exclusion upper bounds are found to be many orders of magnitude higher than $\mathcal{O}\left(10^{-28}\right) \mathrm{cm}^{2}$ obtained from the previous analysis [14]. Our result closes the gap between cross sections excluded by the XENON1T experiment and that by the astrophysical observables such as the CMB, galactic gas cloud cooling and galactic structure formation.

This paper is organized as follows: In Sec. II, we review the CRDM flux calculation formalism and calculate the effective distance using spatial-dependent CR flux and including the contributions from major heavy CR nuclear species. In Sec. III, we analyze Earth attenuation of CRDM using both the analytic approach and numerical simulation to obtain underground CRDM flux. The predicted direct detection recoil spectra and corresponding exclusion regions are discussed in Sec. IV. We conclude this work in Sec. V. 


\section{CRDM FLUX}

\section{A. Kinematics}

In this section we briefly review the basic formalism of CRDM flux calculation. The processes of CR-DM scattering in the galaxy, DM-nucleus scattering in the crust of the Earth and that within the underground detector can be approximated as two-body elastic scattering processes. In the generic case, for an incident particle $X$ which elastically scatters off a fixed target particle $Y$, the recoil kinetic energy $T_{Y}$ of the particle $Y$ is relate to the kinetic energy $T_{X}$ of the particle $X$ as $T_{Y}=T_{Y}^{\max }\left(T_{X}\right)\left(1-\cos \theta^{*}\right) / 2$, where $\theta^{*}$ is the scattering angle in the center-of-mass (CM) frame, and the maximal recoil energy of the target particle $Y$ is given by

$$
T_{Y}^{\max }\left(T_{X}\right)=\left[1+\frac{\left(m_{Y}-m_{X}\right)^{2}}{2 m_{Y}\left(T_{X}+2 m_{X}\right)}\right]^{-1} T_{X} .
$$

The maximal energy transfer occurs when $m_{Y}=m_{X}$, or in the case where the incident particle $\mathrm{X}$ is relativistic with $T_{X} \gg m_{X}^{2} / 2 m_{Y}\left(T_{X} \gg m_{Y} / 2\right)$ for $m_{X} \gg m_{Y}\left(m_{X} \ll m_{Y}\right)$. The minimal energy of the particle $X$ required to produce a recoil energy $T_{Y}$ can be obtained by inverting Eq. (1), which gives

$$
T_{X}^{\min }\left(T_{Y}\right)=\left(\frac{T_{Y}}{2}-m_{X}\right)\left(1 \pm \sqrt{1+\frac{2 T_{Y}}{m_{Y}} \frac{\left(m_{X}+m_{Y}\right)^{2}}{\left(2 m_{X}-T_{Y}\right)^{2}}}\right)
$$

where the $+(-)$ sign applies for the case of $T_{Y}>2 m_{X}\left(T_{Y}<2 m_{X}\right)$. For instance, let us consider a DM direct detection experiment with xenon target nuclei $\left(m_{N}=122 \mathrm{GeV}\right)$ and a detection threshold of $T_{N}^{\mathrm{thr}} \simeq 5 \mathrm{keV}$. For detecting a CRDM particle (denoted as $\chi$ ) with mass $m_{\chi}=1$ (10) $\mathrm{MeV}$ in such an experiment, the required minimal DM kinetic energy, and the corresponding required minimal CR proton kinetic energy are

$$
T_{\chi}^{\mathrm{thr}} \simeq 16.5(10.1) \mathrm{MeV}, \text { and } T_{p}^{\mathrm{thr}} \simeq 1.92(0.22) \mathrm{GeV},
$$

respectively. Thus for detecting $\mathrm{MeV}$ scale DM particles, the required typical CR proton energy is around the GeV scale.

Neglecting the secondary collisions of CRDM with the interstellar medium, the CR boosted DM particles should travel in straight lines before reaching the Earth. The flux (defined as number of particles per unite area, time and solid angle, i.e. $\Phi=d N / d A d t d \Omega$ ) of DM particles up-scattered by CR nuclei species $i$ can be written as [14]

$$
\frac{d \Phi_{\chi}}{d T_{\chi}}=\sum_{i} \int_{\text {l.o.s }} d \ell \frac{\rho_{\chi}(\boldsymbol{r})}{m_{\chi}} \int_{T_{i}^{\min }} d T_{i} \frac{d \sigma_{\chi i}}{d T_{\chi}} \frac{d \Phi_{i}(\boldsymbol{r})}{d T_{i}}
$$

where $\rho_{\chi}(\boldsymbol{r})$ is DM energy density distribution, $m_{\chi}$ is the mass of DM particle, $d \Phi_{i}(\boldsymbol{r}) / d T_{i}$ is the energy distribution of the flux of CR species $i$, and $d \sigma_{\chi i} / d T_{\chi}$ is the differential cross section for the scattering process. The integration of DM flux generated from different positions should be performed along the line-of-sight (l.o.s). 
The angular-averaged CRDM flux is defined as $d \bar{\Phi}_{\chi} / d T_{\chi} \equiv \frac{1}{4 \pi} \int d \Omega\left(d \Phi_{\chi} / d T_{\chi}\right)$, where $\Omega$ is the solid angle. From Eq. (4), the averaged flux can be written as

$$
\frac{d \bar{\Phi}_{\chi}}{d T_{\chi}}=\frac{\rho_{\chi}^{\mathrm{loc}}}{m_{\chi}} \sum_{i} \int d T_{i} K_{i}\left(T_{i}\right) \frac{d \sigma_{\chi i}}{d T_{\chi}} \frac{d \Phi_{i}^{\mathrm{loc}}}{d T_{i}},
$$

where the $K_{i}$ factors which contain all the astrophysical information are defined as

$$
K_{i}\left(T_{i}\right) \equiv \frac{1}{\rho_{\chi}^{\operatorname{loc}} \frac{d \Phi_{i}^{\text {loc }}}{d T_{i}}} \int \frac{d \Omega}{4 \pi} \int_{\text {l.o.s }} d \ell \rho_{\chi}(\boldsymbol{r}) \frac{d \Phi_{i}(\boldsymbol{r})}{d T_{i}}
$$

where the superscript "loc" represents the local value in the solar system. If the energy spectra of the CR flux do not vary significantly within the Galaxy, the spatial dependence of the flux can be factored out as

$$
\frac{d \Phi_{i}(\boldsymbol{r})}{d T_{i}} \approx g_{i}(\boldsymbol{r}) \frac{d \Phi_{i}^{\mathrm{loc}}}{d T_{i}}
$$

In this case, the $K_{i}$ factors become nearly energy independent and only contain the spatial distribution of DM and CR particles.

$$
K_{i} \approx \int \frac{d \Omega}{4 \pi} \int_{\text {l.o.s }} \frac{\rho_{\chi}(\boldsymbol{r})}{\rho_{\chi}^{\text {loc }}} g_{i}(\boldsymbol{r}) d \ell .
$$

Alternatively, the CRDM flux can be rewritten as

$$
\frac{d \bar{\Phi}_{\chi}}{d T_{\chi}}=D_{\mathrm{eff}}\left(T_{\chi}\right) \frac{\rho_{\chi}^{\mathrm{loc}}}{m_{\chi}} \sum_{i} \int d T_{i} \frac{d \sigma_{\chi i}}{d T_{\chi}} \frac{d \Phi_{i}^{\mathrm{loc}}}{d T_{i}},
$$

where the quantity $D_{\text {eff }}\left(T_{\chi}\right)$ is defined as

$$
D_{\mathrm{eff}}\left(T_{\chi}\right) \equiv \frac{\sum_{i} \int d T_{i} K_{i}\left(T_{i}\right) \frac{d \sigma_{\chi i}}{d T_{\chi}} \frac{d \Phi_{i}^{\mathrm{loc}}}{d T_{i}}}{\sum_{i} \int d T_{i} \frac{d \sigma_{\chi i}}{d T_{\chi}} \frac{d \Phi_{i}^{\mathrm{loc}}}{d T_{i}}}
$$

which in general depends on the kinetic energy $T_{\chi}$. In the simplified assumption where the CR flux in the Galaxy is almost the same as the local CR flux, namely, $g_{i}(\mathbf{r}) \approx 1$, all the $K_{i}$ factors become the same, i.e., $K_{i} \approx K$. In this case, the effective distance $D_{\text {eff }}\left(T_{\chi}\right)$ becomes energy independent as well, and reduces to a constant $D_{\text {eff }}\left(T_{\chi}\right) \approx D_{\text {eff }}$ which is given by

$$
D_{\text {eff }} \approx K=\int \frac{d \Omega}{4 \pi} \int_{\text {l.o.s }} \frac{\rho_{\chi}(\boldsymbol{r})}{\rho_{\chi}^{\text {loc }}} d \ell,
$$

which is the so-called effective distance and commonly adopted in the literature [14]. $D_{\text {eff }}=$ $\mathcal{O}(1-10) \mathrm{kpc}$ is often chosen as a benchmark value. The deviation of effective distance $D_{\text {eff }}\left(T_{\chi}\right)$ from a constant directly reflects the effect of the inhomogeneity of the CR flux in CRDM flux. 


\section{B. Calculation of the effective distance}

In most of the recent analyses on CRDM, the value of $D_{\text {eff }}$ was taken as a parameter roughly in the range of $1-10 \mathrm{kpc}$, which becomes a source of uncertainty in phenomenological analysis. This quantity is actually calculable as the CR distribution within the Galaxy is well modeled. In this work, we focus on the CRDM particles with mass around $\mathcal{O}(\mathrm{MeV}-$ $\mathrm{GeV}$ ). This sub-GeV region recently received significant amount of attention as they can be potentially reached by the current DM direct detection experiments with lower thresholds and improved analysis methods. For MeV-GeV scale CRDM particles which are relevant to the DM direct detection, the dominant contribution to their flux comes from CRs with energy below PeV which are mainly of Galactic origin. Our Galaxy is filled with random magnetic fields with typical strength around a few $\mu G$. Charged CR nuclei produced within the Galaxy are trapped by the magnetic fields and in diffusive random motion for a long period of $\mathcal{O}$ (Myrs) before escaping from the Galaxy. The distribution of Galactic CRs are well described by diffusion models with parameters determined by local CR measurements.

The propagation of CR particles through the Galaxy can be approximated by a diffusion model. The diffusion halo is parameterized by a cylinder with radius $R \simeq 20 \mathrm{kpc}$ and halfheight $Z_{\mathrm{h}}=1 \sim 10 \mathrm{kpc}$. The diffusion equation for the $\mathrm{CR}$ charged particles reads [35, 36]

$$
\frac{\partial \psi}{\partial t}=\nabla \cdot\left(D_{x x} \nabla \psi-\boldsymbol{V}_{c} \psi\right)+\frac{\partial}{\partial p} p^{2} D_{p p} \frac{\partial}{\partial p} \frac{1}{p^{2}} \psi-\frac{\partial}{\partial p}\left[\frac{d p}{d t} \psi-\frac{p}{3}\left(\nabla \cdot \boldsymbol{V}_{c}\right) \psi\right]-\frac{1}{\tau_{f}} \psi-\frac{1}{\tau_{r}} \psi+q(\boldsymbol{r}, p),
$$

where $\psi(\boldsymbol{r}, p, t)$ is the CR number density per unit momentum, $D_{x x}$ is the spatial diffusion coefficient, and $\boldsymbol{V}_{c}$ is the convection velocity. The re-acceleration effect is described as diffusion in momentum space and is determined by the coefficient $D_{p p}$. The quantity $d p / d t$ stands for the momentum loss rate. $\tau_{f}$ and $\tau_{r}$ are the time scales for fragmentation and radioactive decay respectively, and $q(\boldsymbol{r}, p)$ is the source term. The convection velocity $\boldsymbol{V}_{c}$ is assumed linearly increases with the distance $z$ from the galactic plane with the gradient $d V_{c} / d z$. The energy-dependent spatial diffusion coefficient $D_{x x}$ is parameterized as $D_{x x}=$ $\beta^{\eta} D_{0}\left(\rho / \rho_{0}\right)^{\delta}$, where $\rho=p / Z e$ is the rigidity of CR particles with electric charge $Z e, \delta$ is the spectral power index which can have different values $\delta=\delta_{1(2)}$ for $\rho$ below (above) a reference rigidity $\rho_{0}$ (4 GV in this work). $D_{0}$ is a normalization constant, $\beta=v / c$ is the velocity of $\mathrm{CR}$ particles and $\eta \approx 0.7$. The momentum diffusion coefficient $D_{p p}$ is related to $D_{x x}$ as $D_{p p} D_{x x}=4 V_{a}^{2} p^{2} /\left(3 \delta\left(4-\delta^{2}\right)(4-\delta)\right)$, where $V_{a}$ is the Alfvén velocity of disturbances in the hydrodynamical plasma [35]. The spatial boundary conditions are set by assuming that free particles escape beyond the halo, i.e., $\psi(R, z, p)=\psi\left(\boldsymbol{r}, \pm Z_{\mathrm{h}}, p\right)=0$. The steady-state solution can be obtained by setting $\partial \psi / \partial t=0$.

We adopt the numerical code GalProp-v54 [37, 38] to solve the CR diffusion equation with given source distributions and boundary conditions. The GalProp code uses real Milky Way information including the gas distribution, magnetic field distribution, etc., and can calculate the $\mathrm{CR}$ species from proton to nickel with related isotopes included. Recently, the HelMod code [39] has been developed to describe the solar modulation and derive the low energy local interstellar (LIS) flux of CR $\bar{p}, e^{-}$, and nuclei $Z \leq 28$ [40]. The HelMod group has performed a fit to the $\mathrm{CR}$ nuclei ${ }_{1} \mathrm{H}-{ }_{28} \mathrm{Ni}$ with $\mathrm{CR}$ data up to $200 \mathrm{TeV}$ from 


\begin{tabular}{ccccc}
\hline \hline$Z_{\mathrm{h}}[\mathrm{kpc}]$ & $D_{0}\left[10^{28} \mathrm{~cm}^{2} \mathrm{~s}^{-1}\right]$ & $\delta$ & $V_{a}\left[\mathrm{~km} \mathrm{~s}^{-1}\right] d V_{\mathrm{c}} / d z\left[\mathrm{~km} \mathrm{~s}^{-1} \mathrm{kpc}^{-1}\right]$ \\
\hline 4.0 & 4.3 & 0.415 & 30 & 9.8 \\
\hline \hline
\end{tabular}

TAB. I: Main propagation parameters of the CR diffusion equation from Ref. [40].

Voyager 1 [41], ACE-CRIS [42], CREAM [43, 44], NUCLEON [45, 46], CALET [47], HEAO3-C2 [48], DAMPE [49], and AMS-02 [50]. The best-fit values of the main propagation parameters are summarized in Tab. I.

The primary source term $q(\boldsymbol{r}, p)$ can be factorized into the product of a spatial and rigidity distribution function, namely, $q_{\text {prim }}(r, z, p) \propto f(r, z) q(R)$. The energy-dependent part takes the form of a smoothly broken power law in rigidity as follows

$$
q(R) \propto\left(\frac{R}{R_{0}}\right)^{-\gamma_{0}} \prod_{i=0}^{2}\left[1+\left(\frac{R}{R_{i}}\right)^{\frac{\gamma_{i}-\gamma_{i+1}}{s_{i}}}\right]^{s_{i}}
$$

where $s_{i}$ is negative (positive) for $\left|\gamma_{i}\right|>\left|\gamma_{i+1}\right|\left(\left|\gamma_{i}\right|<\left|\gamma_{i+1}\right|\right)$. The spectral parameters $R_{i}, \gamma_{i}$ and $s_{i}$ for each CR species are tuned to reproduce the experiment data. Recently, the HelMod group updated the source spectral parameters $[51,52]$ to reproduce the new AMS-02 data of CR iron [53] and fluorine [54]. The primary source spectral parameters are summarised in Appendix B. The spatial distribution of primary source $f(r, z)$ is assumed to follow the standard pulsar distribution $[40,55]$

$$
f(r, z) \propto\left(\frac{r}{r_{\odot}}\right)^{\alpha} \exp \left(-\beta \frac{r-r_{\odot}}{r_{\odot}}-\frac{|z|}{z_{0}}\right)
$$

where $r_{\odot}=8.5 \mathrm{kpc}, \alpha=1.9, \beta=5.0$, and $z_{0}=0.18 \mathrm{kpc}$. The primary source term for each CR species is normalized to the proton source according to their abundances which are provided in Ref. [40]. An overall normalization is introduced after the calculation of $\mathrm{CR}$ propagation to fit the experimental data. We take these two normalization energy at $100 \mathrm{GeV} /$ nucleon with the proton flux at the normalization energy set to $4.47 \times 10^{-9} \mathrm{MeV}^{-1} \mathrm{~cm}^{-2} \mathrm{~s}^{-1} \mathrm{sr}^{-1}$.

For the DM energy distribution, we adopt the standard NFW profile [59] which is parametrized as [60]

$$
\rho_{\chi}^{\mathrm{NFW}}(r)=\rho_{\chi}^{\text {loc }}\left(1+\frac{R_{\odot}}{R_{\mathrm{s}}}\right)^{2}\left(\frac{r}{R_{\odot}}\right)^{-1}\left(1+\frac{r}{R_{\mathrm{s}}}\right)^{-2},
$$

where $R_{\mathrm{s}}=20 \mathrm{kpc}, R_{\odot}=8.5 \mathrm{kpc}$, and $\rho_{\chi}^{\text {loc }}=0.3 \mathrm{GeV} \mathrm{cm}^{-3}$. The variations of CRDM flux derived from different DM density profiles are typically at a few percent level, and choosing the NFW profile leads to more conservative CRDM flux. A detailed comparison of the effective distance of CRDM in different DM density profiles can be found in Appendix A.

In the left panel of Fig. 1, we show the local interstellar (LIS) fluxes of the CR nuclear species of $\mathrm{H}, \mathrm{He}, \mathrm{C}, \mathrm{O}$, and Fe which contribute up to $\sim 78 \%$ of the total CRDM flux for a typical DM particle with $m_{\chi}=10 \mathrm{MeV}$. The LIS fluxes are calculated using GalProp-v54. 

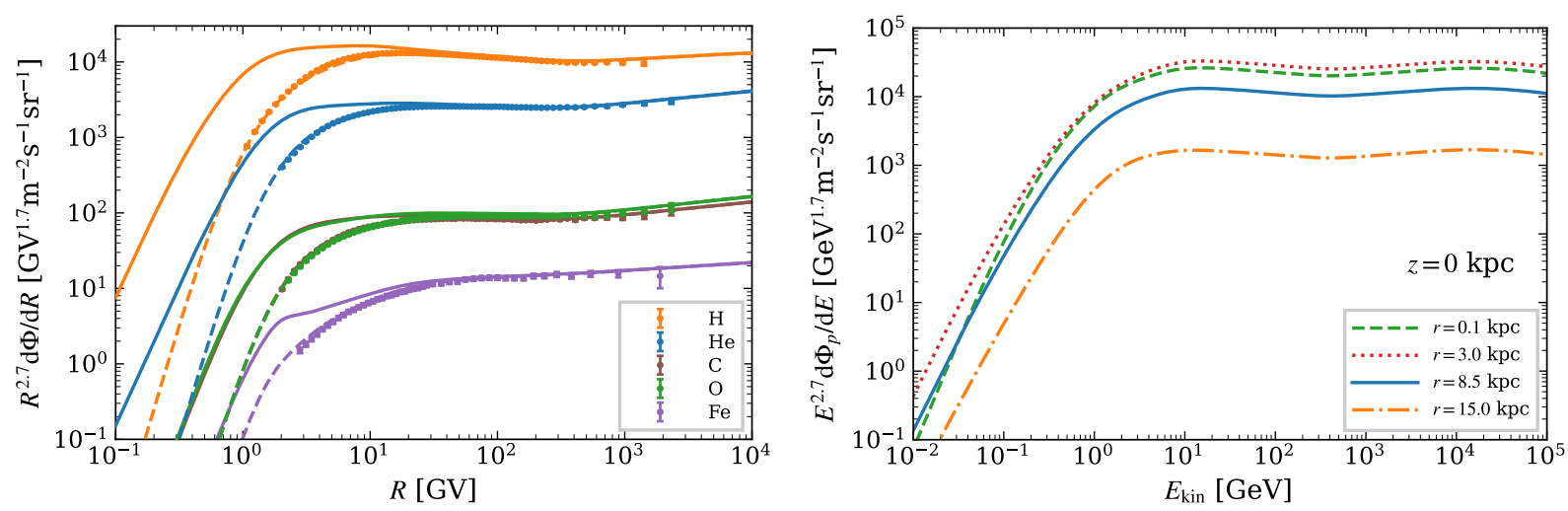

FIG. 1: (Left) CR flux as a function of rigidity for nuclear species of $\mathrm{H}, \mathrm{He}, \mathrm{C}, \mathrm{O}$, and Fe, together with the AMS-02 data [53, 56-58]. The solid lines represent LIS fluxes of CRs calculated using GalProp-v54, and the dashed lines indicate the corresponding solar modulated fluxes calculated using HelMod-v4.1. (Right) CR proton flux as a function of kinetic energy in the galactic plane $(\mathrm{z}=0)$ at different distances to the Galactic Center $r=0.1,3.0,8.5$, and $15.0 \mathrm{kpc}$.

The effect of solar modulation calculated using HelMod-v4.1 are also shown. The results show a good agreement with the local CR measurement such as the recent AMS-02 data. In the right panel of Fig. 1, we show the CR proton fluxes in the galactic plane $(z=$ 0 ) at difference distances to the Galactic Center. It can be seen that all the CR fluxes at different distances have similar spectral shapes, the major differences are only in the overall normalization, which suggests that Eq. (7) is a reasonable approximation for the CR distribution within the Galaxy.

In the left panel of Fig. 2, the values of the $K$ factors calculated from Eq. (6) for the same CR nuclei as that in Fig. 1 are shown. The energy dependencies of the $K$ factors for different $\mathrm{CR}$ species are quite similar: starting from about $4.5 \mathrm{kpc}$ at low energy below $10^{-3} \mathrm{GeV} /$ nucleon, reaching a peak around $10 \mathrm{kpc}$ when the kinetic energy is $\sim 1 \mathrm{GeV} /$ nucleon, and then decreasing to a constant about $8.7 \mathrm{kpc}$ at high energy above $\sim 10 \mathrm{GeV} /$ nucleon. In the energy range of $10^{-3}-10^{3} \mathrm{GeV}$, the overall change in the $K$ factors is about a factor of two. The asymptotic behavior is related to the fact that at high (low) energy region with kinetic energy above $10 \mathrm{GeV} /$ nucleon (below $1 \mathrm{GeV} /$ nucleon) the local and LIS CR spectrum follow the same single power law, thus the energy dependence almost cancels out. Unlike the $K$ factors which are defined purely by astrophysical quantities, the effective distance $D_{\text {eff }}$ essentially depends on the DM-nucleus scattering cross section and DM particle mass. Since the effective distance is an average of $K$ factors weighted by the relevant cross sections and local CR fluxes for a given $T_{\chi}$, the variation in $D_{\text {eff }}$ will not exceed that in the value of the $K$ factors. For large $T_{\chi} \gtrsim \mathrm{GeV}$, the value of $D_{\text {eff }}$ approaches a constant of $D_{\text {eff }} \simeq 9 \mathrm{kpc}$, which is very weakly dependent of $m_{\chi}$.

The CR nucleus-DM scattering cross section $\sigma_{\chi i}$ can be related to that at nucleon level $\sigma_{\chi p}$. In this work we consider spin-independent scattering and adopt the simplified assumption that the DM-nucleon scattering is isospin-conserving. In this case the DM-nucleus scattering cross section at zero momentum transfer $\sigma_{\chi i}^{0}$ is related to DM-nucleon scattering cross section 

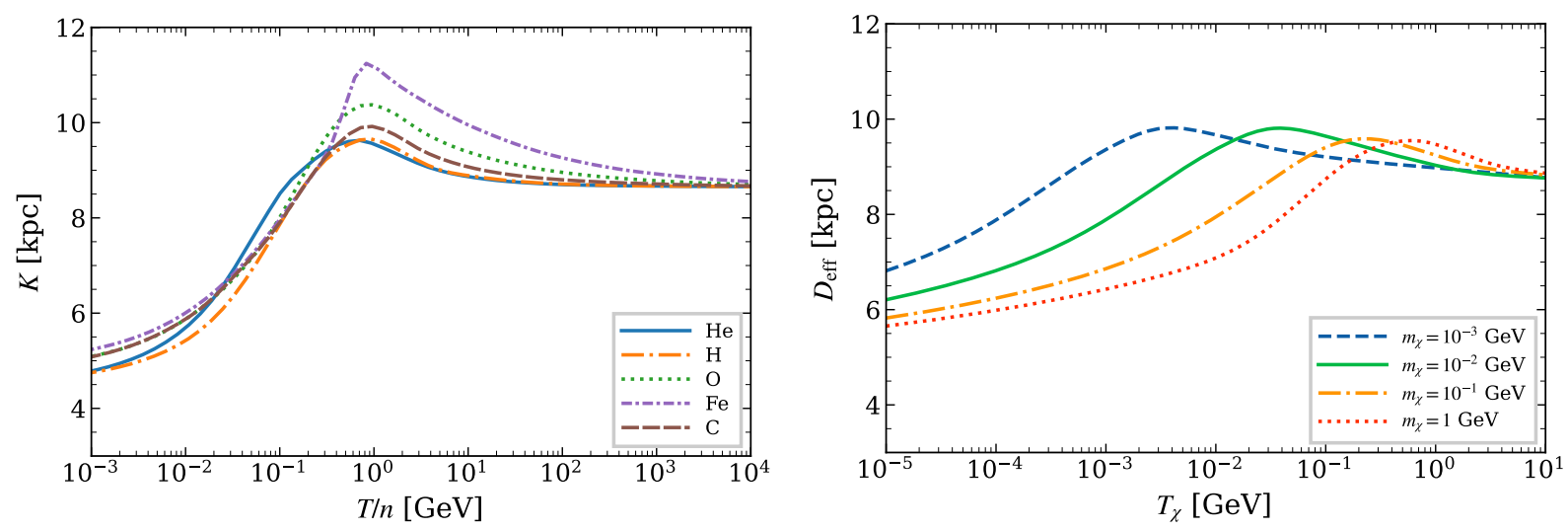

FIG. 2: (Left) $K$ factors as a function of kinetic energy per nucleon for CR nuclei H, He, C, O, and Fe. (Right) Effective distance $D_{\text {eff }}$ as a function of DM kinetic energy with different DM particles masses from $1 \mathrm{MeV}$ to $1 \mathrm{GeV}$.

$\sigma_{\chi p}$ which is to be constrained by DM direct detection experiments as [61]

$$
\sigma_{\chi i}^{0}=A_{i}^{2} \frac{\mu_{\chi i}^{2}}{\mu_{\chi p}^{2}} \sigma_{\chi p},
$$

where $A_{i}$ is the atomic mass number of the nucleus $i$, and $\mu_{a b}=m_{a} m_{b} /\left(m_{a}+m_{b}\right)$ is the two-particle reduced mass, The CR nucleus-DM scattering cross section is given by

$$
\frac{d \sigma_{\chi i}}{d T_{\chi}}=\frac{F_{i}^{2}\left(q^{2}\right) A_{i}^{2}}{T_{\chi}^{\max }\left(T_{i}\right)} \frac{\mu_{\chi i}^{2}}{\mu_{\chi p}^{2}} \sigma_{\chi p},
$$

where $F_{i}\left(q^{2}\right)$ is the nuclear form factor at the momentum transfer $q^{2}=2 m_{\chi} T_{\chi}$. We emphasize that the total cross section $\sigma_{\chi i}$ from integrating Eq. (17) is in general different from $\sigma_{\chi i}^{0}$ after the effect of form factor is taken into account.

\section{CRDM flux}

Using the expression of $T_{i}^{\text {min }}$ in Eq. (2) and the cross section in Eq. (17), the angular averaged flux of CRDM can be calculated as the sum of the contributions from the individual CR species

$$
\frac{d \bar{\Phi}_{\chi}}{d T_{\chi}}=\frac{\rho_{\chi}^{\mathrm{loc}}}{m_{\chi}} \sum_{i} F_{i}^{2}\left(2 m_{\chi} T_{\chi}\right) \sigma_{\chi i}^{0} \int_{T_{i}^{\min }\left(T_{\chi}\right)}^{\infty} d T_{i} \frac{K_{i}\left(T_{i}\right)}{T_{\chi}^{\max }} \frac{d \Phi_{i}^{\mathrm{loc}}}{d T_{i}} .
$$

For proton and helium, we take the dipole form factor $F_{i}\left(q^{2}\right)=1 /\left(1+q^{2} / \Lambda_{i}^{2}\right)^{2}[62]$, where $\Lambda_{p} \approx 770 \mathrm{MeV}$ and $\Lambda_{\mathrm{He}} \approx 410 \mathrm{MeV}$. For heavier nuclei we adopt the conventional Helm form factor $[63,64]$

$$
F\left(q^{2}\right)=\frac{3 j_{1}\left(q R_{1}\right)}{q R_{1}} e^{-\frac{1}{2} q^{2} s^{2}},
$$

where $j_{1}(x)$ is the spherical Bessel function of the first kind, $R_{1}=\sqrt{R_{A}^{2}-5 s^{2}}$ with $R_{A} \approx$ $1.2 A^{1 / 3} \mathrm{fm}$, and $s \approx 1 \mathrm{fm}$. 


\begin{tabular}{lcc}
\hline \hline \multicolumn{2}{c}{ nucleus } & CRDM flux $\left[\mathrm{cm}^{-2} \mathrm{~s}^{-1}\right]$ \\
\hline $\mathrm{He}$ & $1.77 \times 10^{-5}$ & 28.6 \\
$\mathrm{H}$ & $1.38 \times 10^{-5}$ & 22.3 \\
$\mathrm{O}$ & $6.61 \times 10^{-6}$ & 10.7 \\
$\mathrm{Fe}$ & $6.19 \times 10^{-6}$ & 10.0 \\
$\mathrm{C}$ & $3.60 \times 10^{-6}$ & 5.84 \\
$\mathrm{Si}$ & $2.76 \times 10^{-6}$ & 4.48 \\
$\mathrm{Mg}$ & $2.68 \times 10^{-6}$ & 4.35 \\
$\mathrm{Ne}$ & $1.24 \times 10^{-6}$ & 2.01 \\
$\mathrm{~N}$ & $1.07 \times 10^{-6}$ & 1.74 \\
$\mathrm{sum}$ & $5.57 \times 10^{-5}$ & 90.0 \\
\hline \hline
\end{tabular}

TAB. II: Contributions to the total CRDM flux from different CR species. The DM particle mass is fixed at $m_{\chi}=10 \mathrm{MeV}$, and the flux is integrated over the DM kinetic energy range $10^{-5}-10 \mathrm{GeV}$ and $4 \pi$ solid angle. The species which contribute up to $90 \%$ of the total CRDM flux are listed.

In the left panel of Fig. 3, the fluxes of CRDM contributed by the five most dominant CR species are shown and compared with the total flux contributed by all the CR species, for a typical light CRDM particle with $m_{\chi}=10 \mathrm{MeV}$. Among the CR species, helium contribution is the most dominant, which contributes about $28 \%$ of the total CRDM flux. Helium and proton together contribute about 50\% of the total flux. Despite much smaller fluxes, contributions from heavier CR nuclei can be comparable to that from helium/proton due to the enhancement factor $A_{i}^{2}$ in the spin-independent scattering cross section. Including the contributions from oxygen, iron and carbon etc. leads a factor of two enhancement in $D_{\text {eff. }}$ The detailed contribution from each CR species are summarized in Tab. II. Note, however, that for heavier DM particles, the total contribution is still dominated by helium and proton due to the stronger form factor suppression. For instance, for $1 \mathrm{GeV}$ DM particle, the CR proton contributes $47 \%$ of the CRDM flux and helium contributes $39 \%$. During the calculation, we neglect the light CRs lithium and beryllium as they are secondary CRs with very low abundances.

The total CRDM fluxes for different DM particle masses are shown in the right panel of Fig. 3. We compare the CRDM flux of different DM particle masses with that derived using a constant $D_{\text {eff }}=1 \mathrm{kpc}$ [14] in the right panel of Fig. 3. For lighter DM, the flux exhibits a power-law behavior in the region of interest, which is related to the approximate power-law CR spectrum and constant cross section. For heavier DM, the nuclear form factor leads to a steeper decrease in the high-energy part of the flux. The shape of the spectra does not change much after the inhomogeneous CR fluxes are taken into account as $D_{\text {eff }}\left(T_{\chi}\right)$ changes less than a factor of two for the energy range shown in the right panel of Fig. 2. The slope change that can be seen below $10^{-2} \mathrm{GeV}$ for $m_{\chi}=1 \mathrm{GeV}$ is due to the fact that the parameterization of the LIS CR fluxes adopted from [39] are only valid for CRs with rigidity higher than $0.2 \mathrm{GV}$, and the CRDM fluxes contributed by lower energy CRs are ignored. The kinetic energy of the LIS CRs in this work are calculated down to $1 \mathrm{eV}$ per 

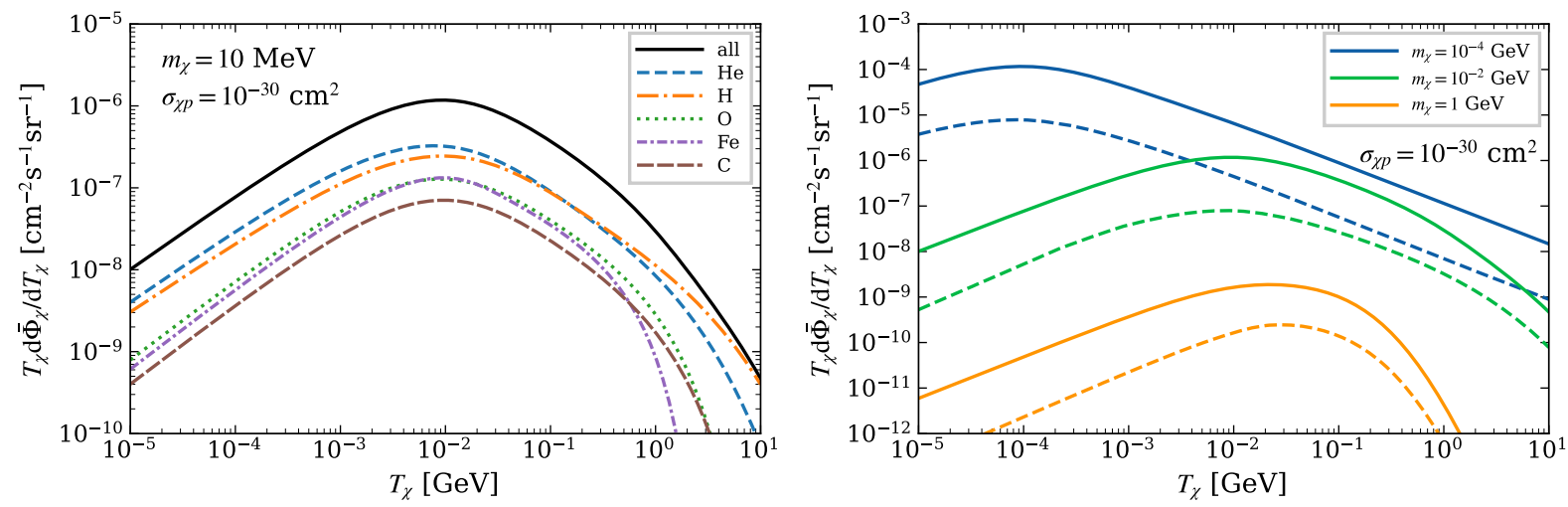

FIG. 3: (Left) CRDM fluxes derived from the five most dominant CR species of $\mathrm{He}, \mathrm{H}, \mathrm{O}, \mathrm{Fe}$, and $\mathrm{C}$ with DM particle mass $m_{\chi}=10 \mathrm{MeV}$ and cross section $\sigma_{\chi p}=10^{-30} \mathrm{~cm}^{2}$. The black solid line indicates the total flux contributed by all the CR species with $Z \leq 28$. (Right) Total CRDM fluxes contributed by all the $\mathrm{CR}$ species for different DM particle masses $m_{\chi}=10^{-4}, 10^{-2}$, and $1 \mathrm{GeV}$ (solid lines) with cross section $\sigma_{\chi p}=10^{-30} \mathrm{~cm}^{2}$. The CRDM fluxes derived from the parameterised LIS fluxes of proton and helium in [39] with a constant effective distance $D_{\text {eff }}=1 \mathrm{kpc}$ in Ref. [14] are also shown for comparison (dashed lines).

nucleon by GalProp-v54. We find that the CRDM fluxes are enhanced by more than an order of magnitude compared with the benchmark model with $D_{\text {eff }}=1 \mathrm{kpc}[14]$. In the whole enhancement, roughly a factor of $\sim 8$ enhancement arises from the value of $D_{\text {eff }}$ and a factor of $\sim 2$ from $C R$ species heavier than proton and helium.

Although in this work we are only interested in the angular-averaged CRDM flux, the CRDM flux at the surface of the Earth is highly anisotropic in the arrival direction. CRDM flux from the direction of the galactic center (GC) is significantly higher than that from the anti-GC (AC) direction. For instance, for DM particles with mass $m_{\chi}=10 \mathrm{MeV}$ and scattering cross section $\sigma_{\chi p}=10^{-30} \mathrm{~cm}^{2}$, the CRDM flux with $T_{\chi} \geq 0.01 \mathrm{MeV}$ and integrated within a $5^{\circ}$ cone around the directions of $\mathrm{GC}$ and $\mathrm{AC}$ are $\Phi_{\mathrm{GC}}=3.1 \times 10^{-6} \mathrm{~cm}^{-2} \mathrm{~s}^{-1}$ and $\Phi_{\mathrm{AC}}=2.6 \times 10^{-8} \mathrm{~cm}^{-2} \mathrm{~s}^{-1}$, respectively. The final anisotropy measured at the underground detectors will be significantly smaller due to the multiscatterings between the DM particles and nucleus within the Earth. The remaining anisotropy can still leads to non-negligible diurnal modulation of the event rate, which can be used to set stringent constraints on the property of CRDM particles [19, 65].

\section{EARTH ATTENUATION}

Before arriving at the underground detectors, CRDM particles lose energy due to the similar elastic scattering off the nuclei in the interstellar gas, the atmosphere and the crust of the Earth. For the cross sections under consideration, the dominant energy-loss process is the scattering off the nuclei within the Earth crust. The energy loss can be estimated using the analytic approach which is based on the one-dimensional collision approximation [66, 67]. 
A more sophisticated approach is to use Monte-Carlo simulations to directly simulate the three-dimensional motion and multiple collisions of DM particles inside the Earth. In this work, both approaches are adopted and compared. We focus on the impact of the form factor on the energy spectrum of CRDM deep underground. For simplicity, we only consider elastic scattering which is an irreducible process.

\section{A. Analytic approach}

In the approximate analytic approach, the decrease of $T_{\chi}$ with respect to depth $z$ due to the elastic scattering off the nucleus $N$ in Earth's crust is given by

$$
\frac{d T_{\chi}}{d z}=-\sum_{N} n_{N} \int_{0}^{T_{N}^{\max }} \frac{d \sigma_{\chi N}}{d T_{N}} T_{N} d T_{N},
$$

where $n_{N}$ is the number density of nucleus species $N$. The maximal recoil energy can be obtained from Eq. (1), and the differential cross section from Eq. (17) using the appropriate substitutions. Since the dominant chemical component of the crust is oxygen with $m_{N} \approx$ $15 \mathrm{GeV}$, we focus on the case where $m_{\chi}, T_{\chi} \ll m_{N}$. Using the expression of $T^{\max }$ in Eq. (1), the energy-loss with respect to the distance to the surface $z$ can be rewritten as

$$
\frac{d T_{\chi}}{d z}=-\frac{1}{\ell_{E}\left(T_{\chi}\right)}\left(T_{\chi}+\frac{T_{\chi}^{2}}{2 m_{\chi}}\right),
$$

where $\ell_{E}$ is the mean free path for energy loss, and $\ell_{E}^{-1}=2 m_{\chi} \sum_{N} g_{N}\left(T_{\chi}\right) n_{N} \sigma_{\chi N}^{0} / m_{N}$. The factor $g_{N}$ in the expression of $\ell_{E}$ which contains the effect of the form factor is given by

$$
g_{N}\left(T_{\chi}\right)=\int_{0}^{T_{N}^{\max }} F_{N}^{2}\left(q^{2}\right) \frac{2 T_{N}}{\left(T_{N}^{\max }\right)^{2}} d T_{N} .
$$

Note that in the generic case the mean free path depends on $T_{\chi}$ through the factor $g_{N}\left(T_{\chi}\right)$.

We start with an oversimplified case where $F_{N}\left(q^{2}\right) \rightarrow 1$. In this case the factor $g_{N}$ approaches unity, as expected. In this simple case the Eq. (21) can be integrated out analytically. The solution for $T_{\chi}(z)$ at depth $z$ is related to that at surface $T_{\chi}(0)$ as follows

$$
T_{\chi}(z)=\frac{T_{\chi}(0) e^{-z / \ell_{E}^{0}}}{1+\frac{T_{\chi}(0)}{2 m_{\chi}}\left(1-e^{-z / \ell_{E}^{0}}\right)},
$$

where $\ell_{E}^{0}$ is the mean free path for energy loss in the case of $F_{N}\left(q^{2}\right)=1$. For isospinconserving scattering, $\left(\ell_{E}^{0}\right)^{-1} \approx 2 m_{\chi} \rho_{\oplus} \sigma_{\chi p} / m_{p}^{2}$ where $\rho_{\oplus}=2.7 \mathrm{~g} \cdot \mathrm{cm}^{-3}$ is the averaged mass density of the outer crust of the Earth, and $m_{p}$ is the proton mass. Note that in this special case $\ell_{E}^{0}$ is independent of the chemical composition of the crust [24].

For relativistic CRDM particles, the energy loss term in Eq. (21) is dominantly proportional to $T_{\chi}^{2}$. Thus for higher energy DM particles, the energy loss is more efficient. Due to the fast energy loss, if the CRDM particles remain to be relativistic at depth $z$ with 
$z \ll \ell_{E}^{0}$, the CRDM flux will be cutoff at a maximal energy as suggested by Eq. (23). For isospin-conserving scatterings, the cutoff energy can be approximated as

$$
T_{\chi}^{\text {cut }}(z) \approx \frac{m_{p}^{2}}{z \rho_{\oplus} \sigma_{p}} .
$$

Note that it is independent of the initial value of $T_{\chi}(0)$ at the surface and the DM particle mass. For a given experiment with a detection threshold $T_{N}^{\text {thr }}$, there exists a threshold in the DM energy $T_{\chi}^{\text {thr }}$ below which the DM particle cannot register a recoil signal. If $T_{\chi}^{\text {cut }}(z)$ is below this threshold, then the DM particles become undetectable. From this cutoff, one can set a upper bound on the cross section $\sigma_{\chi p}$ that can be reached by the experiment under consideration. Using the benchmark values given in Eq. (3), for $z=1.4 \mathrm{~km}$, the upper bounds is $\sigma_{\chi p} \sim 2.5 \times 10^{-28} \mathrm{~cm}^{2}$ which is highly insensitive to the DM particle mass. This result is in an excellent agreement with the findings in the previous analysis in [14]. If the CRDM particles become non-relativistic at depth $z$. The cutoff will mildly depend on the DM initial kinetic energy $T_{\chi}(0)$ at the surface, and the DM energy decreases with depth exponentially, i.e., $T_{\chi}(z) \propto T_{\chi}(0) e^{-z / \ell_{E}}$.

In the next step, we turn on the effect of nuclear form factors. For CRDM particles with energy above $\sim \mathrm{GeV}$, the typical momentum transfer $q$ in the scattering off the nuclei within the crust can easily be above a few tens of $\mathrm{MeV}$, which suggests that for high energy CRDM, the total cross section $\sigma_{\chi N}$ can be significantly smaller than $\sigma_{\chi N}^{0}$. The ratio $R$ between the two cross sections is given by

$$
R\left(T_{\chi}\right) \equiv \frac{\sigma_{\chi N}}{\sigma_{\chi N}^{0}}=\int_{0}^{T_{N}^{\max }\left(T_{\chi}\right)} \frac{F^{2}\left(q^{2}\right)}{T_{N}^{\max }\left(T_{\chi}\right)} d T_{N},
$$

where $q^{2}=2 m_{N} T_{N}$. For typical values of $T_{\chi}=100 \mathrm{MeV}, 1 \mathrm{GeV}$ and $10 \mathrm{GeV}$, the corresponding ratios with $m_{\chi}=10 \mathrm{MeV}$ and $m_{N} \approx 15 \mathrm{GeV}$ for oxygen are $R=0.40,5.7 \times 10^{-3}$ and $1.2 \times 10^{-4}$, respectively. Thus for CRDM with energy above GeV, the total cross section is suppressed by more than two orders of magnitude. Consequently, the energy loss due to the Earth attenuation can be much smaller.

The Helm form factor in Eq. (19) is in general an oscillating function. However, before reaching the first zero of the Bessel function $\zeta_{1}=4.449$, i.e, $q R_{1} \leq \zeta_{1}$, the Helm form factor can be well approximated as a Gaussian form factor

$$
F\left(q^{2}\right)_{N} \approx e^{-q^{2} / \Lambda_{N}^{2}} \quad\left(\text { for } q R_{1}<\zeta_{1}\right),
$$

where $\Lambda_{N}^{-2} \approx R_{1}^{2} / a^{2}+s^{2} / 2$ and $a \approx 3.2$. For oxygen nuclei $\Lambda_{N} \approx 0.207 \mathrm{GeV}$. In this case, the evolution equation can be approximated as

$$
\frac{d T_{\chi}}{d z} \approx-\frac{1}{2} \sum_{N} n_{N} \sigma_{\chi N}^{0} T_{N}^{\max } f\left(4 m_{N} T_{N}^{\max } / \Lambda_{N}^{2}\right),
$$

where the function $f(x)=2\left[1-(1+x) e^{-x}\right] / x^{2}$ describes the effect of the form factor. It is evident that $f(x) \rightarrow 1$ for $x \rightarrow 0$. For large $x$, the function has the asymptotic behavior of 


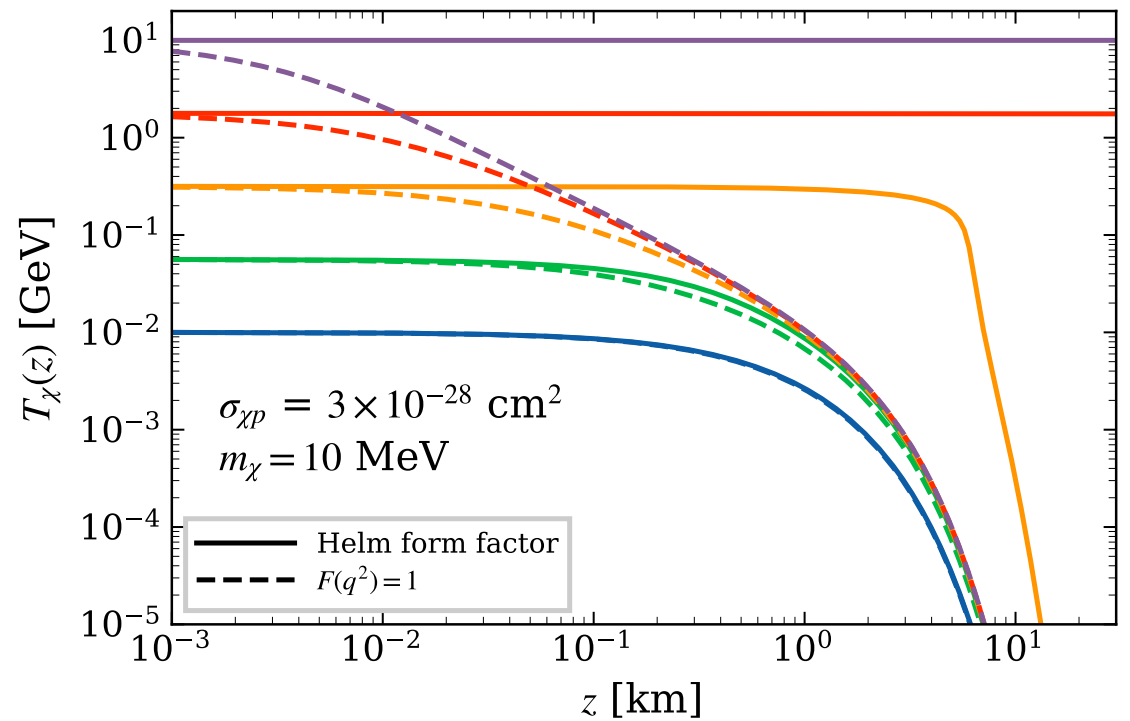

FIG. 4: Solution of the energy loss equation of Eq. (20) as a function of depth $z$ for different initial DM kinetic energies at the surface of the Earth $(z=0)$. The DM particle mass and the cross section are set to be $m_{\chi}=10 \mathrm{MeV}$ and $\sigma_{\chi p}=3 \times 10^{-28} \mathrm{~cm}^{2}$, respectively. The results calculated with the effect of the nuclear form factor considered (neglected) are shown with solid (dashed) lines.

$f(x) \rightarrow 2 / x^{2}$. Thus the solution for large $x$ is

$$
T_{\chi}^{3}(z) \approx T_{\chi}^{3}(0)-\frac{3 z}{32} \sum_{N} n_{N} \sigma_{\chi N}^{0} \frac{\Lambda_{N}^{4}}{m_{N}} .
$$

The second term in the r.h.s of the above equation is a relatively small quantity. It is less than $(0.15 \mathrm{GeV})^{3}$ for $\sigma_{\chi p} \leq 10^{-28} \mathrm{~cm}^{2}$ at $z=1.4 \mathrm{~km}$. Thus in a good approximation $T_{\chi}(z) \approx T_{\chi}(0)$ for CRDM particles with energy above $\mathrm{GeV}$, which suggests that energy loss is negligible for high energy CRDM particles.

The values of the underground CRDM kinetic energy $T_{\chi}(z)$ as a function of depth $z$ from numerically solving the Eq. (20) with the Helm form factor included are shown in Fig. 4 for different initial values of $T_{\chi}(0)$ at surface. For simplicity, we use the uniform density Earth model as in Ref. [68], with the relative chemical element abundances listed in Tab. III. In the figure we consider a typical DM particle mass $m_{\chi}=10 \mathrm{MeV}$ and cross section $\sigma_{\chi p}=3 \times 10^{-28} \mathrm{~cm}^{2}$. The analytic solutions without including the form factor from Eq. (23) are also shown for comparison. For small initial value $T_{\chi}(0) \lesssim 0.1 \mathrm{GeV}$, the two approaches agree with each other quite well. The CRDM energy drops rapidly after $z \gtrsim 1 \mathrm{~km}$. However, for higher energy CRDM particles, the effect of the form factor becomes significant. Including the form factor essentially reduces the total cross section, which result in smaller energy loss. For CRDM above GeV, the energy loss is so small that the DM particle energy is almost unchanged at depth $z \sim \mathrm{km}$. The numerical results in Fig. 4 confirm the approximate analytic result in Eq. (28). 


\section{B. Monte Carlo simulation}

In the analytic method discussed above, the changes in the direction of CRDM particles after each scattering process and the possibility of multiple scatterings are not taken into account, which could lead to inaccurate description of the CRDM energy loss process. This problem becomes serious for CRDM particles with mass and energy significantly lower than the mass of the nucleus. In this case the actual trajectory of the CRDM particle can be much longer than that estimated using the straight-line propagation approximation. A more accurate estimation on the energy loss can be obtained using numerical Monte Carlo (MC) simulations.

In recent years, a number of public codes are developed for the Earth attenuation of non-relativistic halo DM. The codes based on analytic method include EarthShadow [69] which takes into account DM deflection based on single scattering process, and VERNE [70] which considers DM particles travelling on straight-line trajectories. Both of the codes take into account the three-dimensional geometry of the Earth which allows for the study of diurnal modulation. The attenuation of CRDM based on Eq. (20) is also implemented in DarkSUSY-v6.2 [71] with the nuclear form factor neglected. The codes based on MC simulation such as DAMATIS [72] takes a flat Earth model and tracks the vertical distance of the particles only. More sophisticated simulations are implemented in the DaMaSCUS-CRUST code for non-relativistic halo DM [68] which models the overburden as planar layers. In the DaMaSCUS code [73] a preliminary reference Earth model is implemented [74] and a realistic three dimensional halo DM injection is applied. Recently, the realEarthScatterDM code was developed to investigate the daily modulation feature of the boosted electrophilic DM [75], which also considered the density profile of the Earth and focused on the scattering between DM and bound state electrons.

Since most of the current packages focus on non-relativistic halo DM, in this work we develop an alternative simulation code DarkProp [34] which aims at an unified analysis framework for the Earth attenuation of both the relativistic and non-relativistic DM particles. We follow the approach where the free-propagation length is sampled using the mean-free-path calculated from the total cross section $\sigma_{\chi N}$ from integrating Eq. (17) rather than $\sigma_{\chi N}^{0}$. The distribution of the scattering angle after the scattering is sampled according to the differential cross section Eq. (17) and appropriately normalized. The chemical composition of the crust is also taken is to account. The condition for the termination of each DM trajectory is that the DM particle finally leaves the surface of the Earth, or the kinetic energy is less than a given threshold which cannot trigger a recoil signal. This threshold energy is conservatively set to be $10^{-5} \mathrm{GeV}$ for all the DM particle masses considered in this work. Note that our approach is different from that in the DAMATIS code and Ref. [16] where the simulation stops when the particle reaches the depth of the detector. This approach

\begin{tabular}{ccccccccc}
\hline \hline Element & ${ }^{16} \mathrm{O}$ & ${ }^{28} \mathrm{Si}^{27} \mathrm{Al}$ & ${ }^{56} \mathrm{Fe}^{40} \mathrm{Ca}$ & ${ }^{39} \mathrm{~K}$ & ${ }^{23} \mathrm{Na}$ & ${ }^{24} \mathrm{Mg}$ \\
\hline [wt\%] & 46.6 & 27.7 & 8.1 & 5.0 & 3.6 & 2.8 & 2.6 & 2.1 \\
\hline \hline
\end{tabular}

TAB. III: The chemical composition of the Earth's crust from Ref. [68]. 

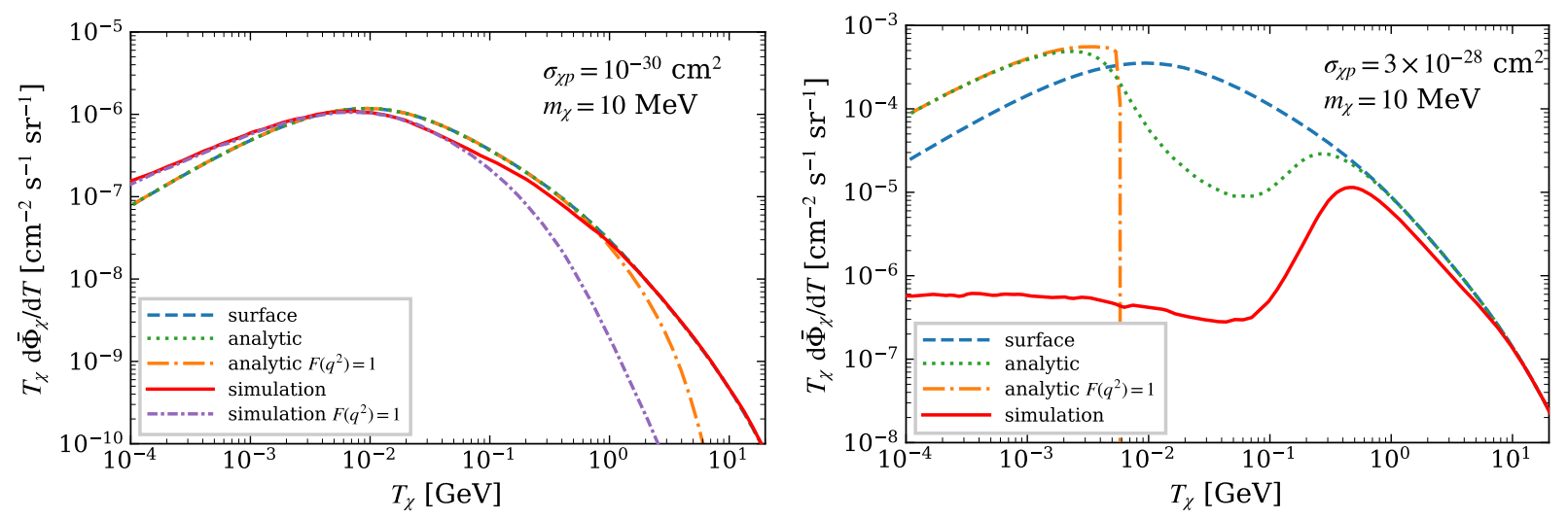

FIG. 5: Underground CRDM flux at a depth of $1.4 \mathrm{~km}$ as a function of kinetic energy with mass $m_{\chi}=10 \mathrm{MeV}$ derived from different approaches for two cross sections $\sigma_{\chi p}=10^{-30} \mathrm{~cm}^{2}$ (left) and $3 \times 10^{-28} \mathrm{~cm}^{2}$ (right). The fluxes at the surface of the Earth are indicated as blue dashed lines. The green dotted lines (orange dash-dotted lines) stand for CRDM fluxes calculated by the analytic method with the nuclear form factor considered (neglected). The red solid lines represent the CRDM fluxes calculated using the MC simulation with the form factor taken into account. The result of the MC simulation with the form factor neglected is not shown in the right panel, as the flux is too low due to the strong attenuation.

underestimates the underground flux and will lead to very conservative estimations of the exclusion region of the DM-nucleon scattering cross section. The details of the algorithms and implementations are summarized in Appendix C.

In this work, for simplicity, we adopted a homogeneous spherical Earth model with density and chemical composition the same as that used in our analytic analysis. Although the densities of the core and the mantle are larger than that of the outer crust, the difference has little effect on the exclusion region. A more realistic Earth model should be relevant for the study of the diurnal effect. We perform simulations of the DM propagation inside the Earth to obtain the underground DM flux in the energy range of interest. This is necessary as light DM particles can enter and leave a given surface within the Earth multiple times due to the relatively large deflection angle.

As a cross check, we find that our code well-reproduces the results of the DaMaSCUS code for non-relativistic halo DM especially the shielding effect at large cross section [68]. Details on the comparison between the two codes can be found in Appendix D.

\section{Underground CRDM flux}

In Fig. 5, we show the underground CRDM fluxes at a depth of $z=1.4 \mathrm{~km}$ for a representative DM particle mass $m_{\chi}=10 \mathrm{MeV}$ and cross section $\sigma_{\chi p}=3 \times 10^{-28} \mathrm{~cm}^{2}$ calculated using four different approaches: analytic approach and MC simulation with and without including the Helm form factor. For the analytic method, the attenuated DM flux 
at depth $z$ is related to that at surface through the relation

$$
\frac{d \Phi}{d T_{\chi}(z)}=\frac{d \Phi}{d T_{\chi}(0)} \frac{d T_{\chi}(0)}{d T_{\chi}(z)},
$$

where the solution of the energy loss equation Eq. (20) and surface CRDM flux obtained in Eq. (4) are adopted. For MC simulation, we simulated $\mathcal{O}\left(10^{7}\right)$ DM particles drawn from surface CRDM flux with kinetic energy between $10^{-5}$ and $100 \mathrm{GeV}$. Then the underground flux is produced using the method detailed in Appendix C.

In Fig. 5, the underground CRDM fluxes at depth $z=1.4 \mathrm{~km}$ calculated using different approaches for two typical cross sections are compared. The DM particle mass is fixed at $m_{\chi}=10 \mathrm{MeV}$. In the left panel, we consider the case with $\sigma_{\chi p}=10^{-30} \mathrm{~cm}^{2}$ which corresponds to relatively small Earth attenuation. In this case, after the form factor is included, both the analytical approach and $\mathrm{MC}$ simulation predict that the underground CRDM flux is almost the same as that at the surface. While the methods neglecting the form factor underpredict the DM flux at high energies above $\sim 1 \mathrm{GeV}$. In the right panel, we consider the case with a relatively large cross section of $\sigma_{\chi p}=3 \times 10^{-28} \mathrm{~cm}^{2}$. The analytic approach with form factor neglected predicts a sharp cutoff in the CRDM flux at kinetic energy $6 \times 10^{-3} \mathrm{GeV}$ in accordance with Eq. (24). Due to this apparent cutoff, the CRDM should not register a signal at a typical xenon based detector with a threshold around keV, thus forming a detection "blind spot" for DM detection [14, 23-25]. After the form factor is turned on, the situation changes dramatically. Both the analytic approach and the MC simulation predict that the CRDM flux can extend to much higher energy regions and finally approach the surface flux for DM energy above GeV, which suggests that for such a large cross section the underground DM can still be energetic enough.

Compared with the analytic approach, the MC simulation predicts smaller CRDM flux by a few orders of magnitude at low energy below $100 \mathrm{MeV}$, which is related to the multiple scattering processes during Earth attenuation. The ordinary mean-free-path $\ell$ which characterizes the average free propagation distance between two successive scatterings (see Eq. (C2) in Appendix) is actually quite small for the large cross section of $3 \times 10^{-28} \mathrm{~cm}^{2}$ considered in the right panel of Fig. 5. In this case, it is found to be $\ell \simeq 8.2 \times 10^{-4} \mathrm{~km}$ for low energy DM particles, which suggests that the DM particle should go through multiple scatterings before reaching the underground detector of XENON-1T. Such multi-scattering process cannot be well described by the analytical approach (green dotted). MC simulation (red solid) is more suitable, as it takes into account the directional change of DM particle in each scattering. A consequence of the multi-scattering is that the actual distance traveled by the DM particles can be much longer than the depth of the detector $z=1.4 \mathrm{~km}$. Thus the energy loss is much more significant than that estimated from the analytical approach. This leads to further distortion of the underground DM energy spectrum. Note that DM particles with lower energies suffer from larger energy losses as the corresponding cross sections are less form-factor suppressed. As it can be seen from the right panel of Fig. 5, compared with the result from the analytic approach, the numerical MC simulation show more significant distortion in the lower energy region. The underground CRDM particles with $T_{\chi} \lesssim 0.1 \mathrm{GeV}$ come dominantly from the energy-loss of the DM particles in 
the narrow energy range $0.1-0.5 \mathrm{GeV}$ on the surface, which roughly follows a power-law $d \Phi_{\chi} / d T_{\chi} \propto T_{\chi}^{-2}$. Due to the spectrum distortion the underground DM flux changes to roughly $d \Phi_{\chi} / d T_{\chi} \propto T_{\chi}^{-1}$ which looks approximately like a flat distribution after rescaled by $T_{\chi}$ in Fig. 5.

In summary, these results show that the effect of the nuclear form factor is significant in the Earth attenuation. It suppresses the attenuation of high-energy CRDM regardless of whether the analytic method or the MC simulation is used. In addition, the MC simulation always leads to a stronger attenuation than the analytic approximation for the mass range under consideration.

\section{CONSTRAINTS FROM DIRECT DETECTION EXPERIMENT}

\section{A. Recoil spectrum}

Using the underground CRDM flux at depth $z$, the differential nuclear recoil event rate per target nucleus mass in the direct detection experiments can be calculated as

$$
\Gamma_{N}=\frac{4 \pi}{m_{N}} \int_{T_{\chi}^{\min }}^{\infty} \frac{d \sigma_{\chi N}}{d T_{N}} \frac{d \bar{\Phi}_{\chi}}{d T_{\chi}} d T_{\chi},
$$

where $m_{N}$ is the mass of the target nucleus $N$ in the detector. In this work, we focus on the XENON1T experiment, which is a xenon dual-phase time projection chamber. For $\mathrm{XENON} 1 \mathrm{~T}, m_{N} \simeq 122 \mathrm{GeV}$. The form factor in the differential cross section takes the same form as that the calculation of Earth attenuation.

In Fig. 6, the recoil event spectrum for the large cross section case corresponds to the same parameters in the right panel of Fig. 5 are shown. The results show that with the form factor included, both the analytic approach and the MC simulation predict signals in the XENON1T S1-S2 signal region of interest (ROI) of $4.9-49 \mathrm{keVnr}$. The rapid decreasing and oscillation of the event rate above the ROI are due to the properties of the Helm form factor.

\section{B. Exclusion regions}

Since the nuclear recoil event rate from the collisions with CRDM is quite different from that with the non-relativistic halo DM, to be more accurate on the effect of threshold, we derive the limits directly from the distribution of the signals, rather than naively rescaling the reported experimental limits from halo WIMP searches [14, 20, 21]. The theoretical recoil event rate needs to be converted into signals according to the signal response model of the specific experiment before it can be compared with the experimental data. Our analysis is base on the S1-S2 data of the XENON1T experiment [76]. The XENON1T experiment utilizes the liquid xenon time projection chambers to detect the recoil energy of the target nuclei from their scattering with DM particles. The deposited energy can produce a prompt scintillation signal (S1) and ionization electrons which are extracted into gaseous xenon 


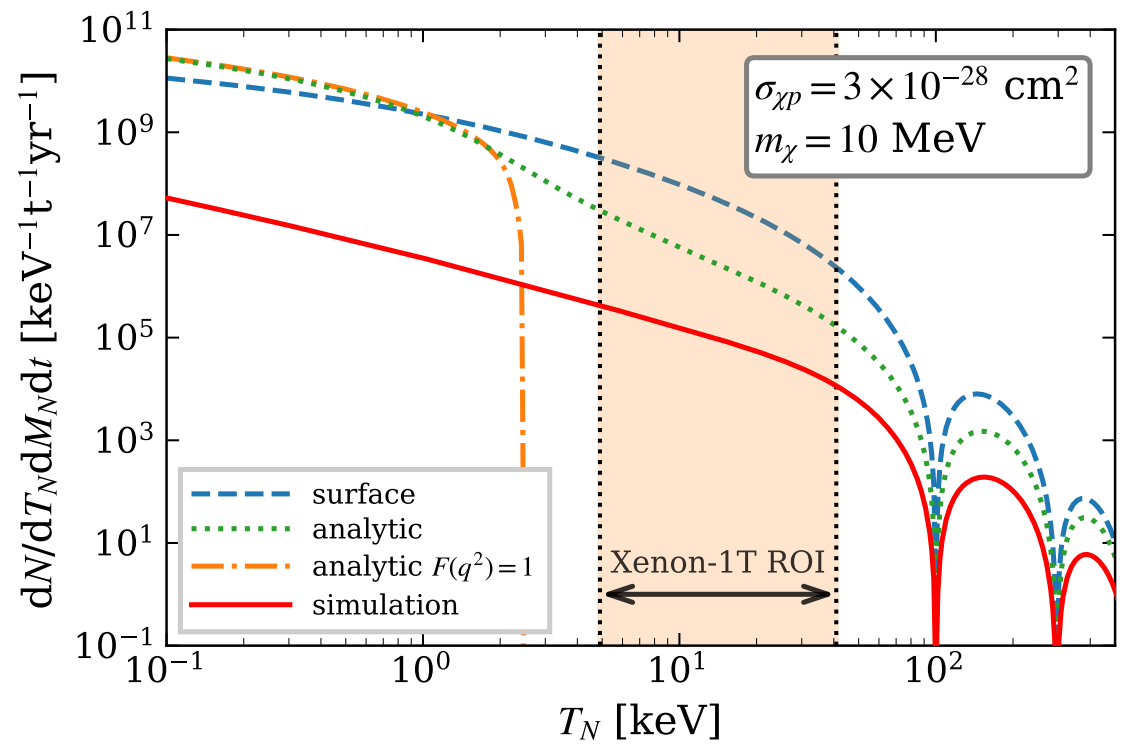

FIG. 6: Recoil event rates of the xenon target nuclei from elastic scattering with CRDM particles at surface and underground corresponding to the CRDM flux in the right panel of Fig. 5 The regionof-interest (ROI) $4.9-40.9 \mathrm{keVnr}$ considered by the XENON1T S1-S2 data analysis is indicated with vertical black dotted lines.

and produce proportional scintillation light (S2). The signal size ratio of S2/S1 allows for discrimination between nuclear and electronic recoils. The S1 and S2 signals will be corrected for their spatial dependence, which result in the corrected signals of $c S 1$ and $c S 2_{b}$ (corresponding to the S2 signals from the bottom photomultiplier tubes). The detailed signal response model can be found in Ref. [77]. We numerically calculate the signal distributions of the scattering between CRDM particles and target xenon nuclei, and derive the excluded regions in $\left(m_{\chi}, \sigma_{\chi p}\right)$ plane for the XENON1T data (S1-S2) [1, 78] using the binned Poisson statistic approach $[79,80]$. The distribution of the background events are taken from the XENON1T analysis. The calculation procedure of the binned Poisson statistic approach is the same as that in our previous work [24].

The exclusion regions at $90 \%$ C.L. calculated by different methods are compared in Fig. 7. For each DM particle mass, we scan the values of the scattering cross section to find the boundaries of the exclusion region. We find that the exclusion lower bound reaches $\sim$ $4 \times 10^{-32} \mathrm{~cm}^{2}$ for $\mathrm{MeV}$ scale DM, which is a factor of five improvement compared with that derived under the assumption of $D_{\text {eff }}=1 \mathrm{kpc}$ [14]. For the cross sections below the exclusion lower bounds, although the downward CRDM particles reaching the depth of the detector do not suffer from much energy loss, the upward CRDM particles entering from the opposite side of the Earth can still loss considerable amount of energy. If the anisotropy of the CRDM flux in the arrival direction is considered, there should be a daily modulation of the underground DM flux, which can be used to explore CRDM in the future [19].

In the case of neglecting the nuclear form factor, the exclusion upper bound obtained from the analytic approach is quite low $\sim 2 \times 10^{-28} \mathrm{~cm}^{2}$, which is as expected. After 


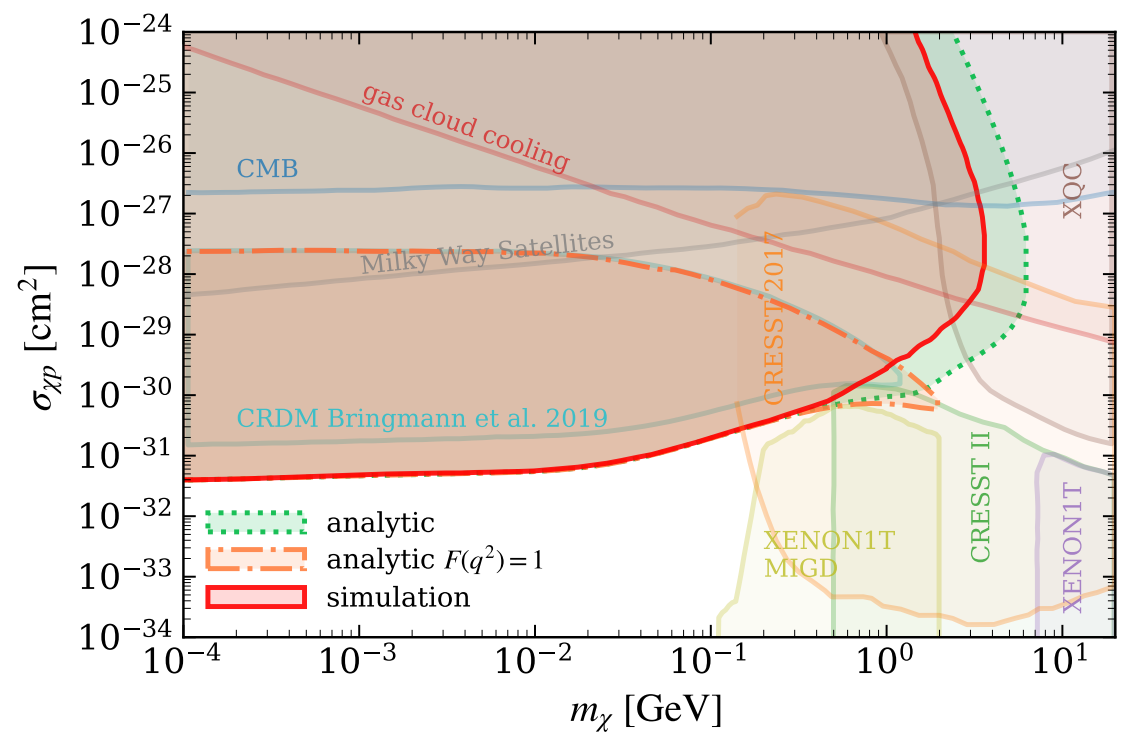

FIG. 7: Exclusion regions in the $\left(m_{\chi}, \sigma_{\chi p}\right)$ plane at $90 \%$ C.L. for CRDM derived from the XENON1T S1-S2 data. The red solid contour represent the exclusion region calculated by the MC simulation with the nuclear form factor considered. The result using the analytic method with the form factor considered (neglected) is shown with green dotted (orange dash-dotted) contours. The lower bounds of these three contours overlap for DM particle mass $m_{\chi} \lesssim 0.2 \mathrm{GeV}$ due to the negligible Earth attenuation in this region. A selection of constraints from other direct detection experiments [81-84] with Earth attenuation considered [68], XQC [85], CMB [32], gas cloud cooling [8], and Milky Way satellite [86] are shown for comparison. The exclusion region derived with a constant effective distance $D_{\text {eff }}=1 \mathrm{kpc}$ in Ref. [14] is also shown.

the form factor is consistently included, we find that the exclusion upper bounds can be increased at least by four orders of magnitude. The bounds from the MC simulation is again more conservative than that from the analytic approximation. This new exclusion region, derived from MC simulation with nuclear form factor considered, is complementary to other constraints derived from astrophysical and cosmological observables. It also overlaps with the exclusion regions reported from DM direct detection experiments with low energy thresholds, especially the CRESST 2017 surface run. Those limits however, is affected by the assumption of the DM halo velocity distribution. Especially the value of the DM escape velocity. On the other hand, the halo velocity distribution has basically no effect on the detection of CRDM particles.

\section{CONCLUSIONS}

In summary, we have performed a refined analysis on the exclusion bounds of the DMnucleon scattering cross section $\sigma_{\chi p}$ using CR boosted DM. We have determined the effective distance $D_{\text {eff }}$ using spatial-dependent CR fluxes and included the contributions from all the major CR nuclear species. We obtained $D_{\text {eff }} \simeq 9 \mathrm{kpc}$ for CRDM particle with kinetic 
energy above $\sim 1 \mathrm{GeV}$, and found that the corresponding exclusion lower bounds can be as low as $\sigma_{\chi p} \sim 4 \times 10^{-32} \mathrm{~cm}^{2}$ for DM particle mass around $\mathrm{MeV}$ scale and below. We have simulated the effect of Earth attenuation with the nuclear form factor fully taken into account. Using both the analytic and numerical approaches, we have shown that for the CRDM particles with mass below GeV scale, the presence of the nuclear form factor strongly suppress the effect of Earth attenuation. Consequently, the cross section that can be excluded by the XENON1T experiment can be many orders of magnitude higher, which closes the gap in the cross sections excluded by the XENON1T experiment and that by the astrophysical observables such as the cosmic microwave background (CMB) and galactic gas cloud cooling, etc.. In this work we have considered energy-independent cross sections. It is straightforward to extend the current framework to energy-dependent cross sections. Further improvements that can be made is to consider the anisotropy of CRDM and the diurnal modulation effect in the future.

\section{Acknowledgments}

This work is supported in part by the National Key R\&D Program of China No. 2017YFA0402204, the Key Research Program of the Chinese Academy of Sciences (CAS), Grant NO. XDPB15, the CAS Project for Young Scientists in Basic Research YSBR-006, and the National Natural Science Foundation of China (NSFC) No. 11825506, No. 11821505, No. 12047503.

\section{Appendix A: Comparison of Dark Matter Profiles}

In this section, we consider the uncertainties in the CRDM flux from different DM density profiles. In particular, we compare the effective distances $D_{\text {eff }}\left(T_{\chi}\right)$ defined in Eq. (10)

predicted with different DM profiles, as the flux of CRDM is directly proportional to this quantity.

In addition to the NFW profile in Eq. (15) considered in the main text, we consider the Einasto profile [87] which is a typical "Cuspy" profile, and the Isothermal profile [88] which is "Cored", for comparison purpose. The Einasto profile is parameterised as [87]

$$
\rho_{\chi}^{\mathrm{EIN}}(r)=\rho_{\chi}^{\mathrm{loc}} \exp \left[-\left(\frac{2}{\alpha}\right)\left(\frac{r^{\alpha}-R_{\odot}^{\alpha}}{R_{\mathrm{s}}^{\alpha}}\right)\right],
$$

where $\rho_{\chi}^{\text {loc }}=0.3 \mathrm{GeV} / \mathrm{cm}^{3}, \alpha=0.17, R_{\odot}=8.5 \mathrm{kpc}$, and $R_{\mathrm{s}}=20 \mathrm{kpc}$. The Isothermal profile is parameterised as [60]

$$
\rho_{\chi}^{\mathrm{ISO}}(r)=\rho_{\chi}^{\mathrm{loc}} \frac{R_{\odot}^{2}+R_{\mathrm{c}}^{2}}{r^{2}+R_{\mathrm{c}}^{2}},
$$

where $R_{\mathrm{c}}=2.8 \mathrm{kpc}$. These DM density profiles are shown in the left panel of Fig. 8 .

For a given DM particle mass $m_{\chi}=10 \mathrm{MeV}$, we repeat the calculations in Sec. II B using different DM density profiles. The resulting effective distances are shown in the right 

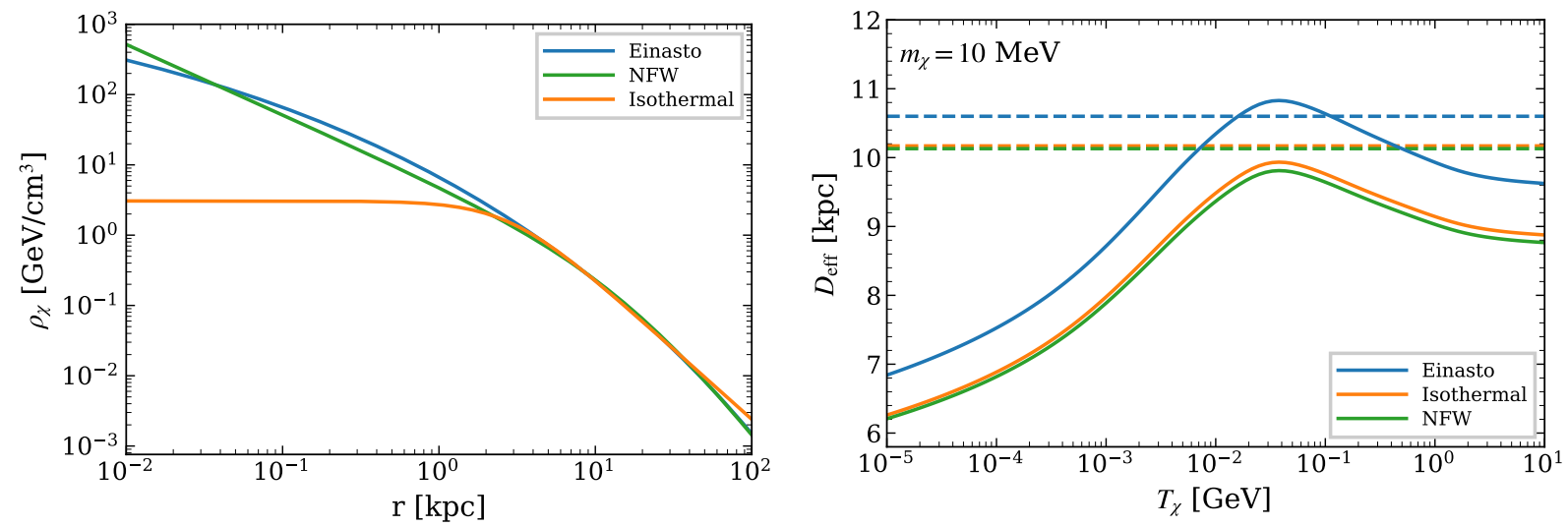

FIG. 8: (Left) Einasto, NFW, and Isothermal DM density profiles as a function of the radial distance from the Galactic Center. (Right) Effective distance of CRDM derived from different DM density profiles of Einasto, Isothermal, and NFW (solid lines). The DM particle mass is fixed at $m_{\chi}=10 \mathrm{MeV}$, and the CR fluxes are the same as that used in Fig. 2. The horizontal dashed lines represent the corresponding $D_{\text {eff }}$ assuming a homogeneous CR distribution.

panel of Fig. 8. We find that the Einasto profile leads to the largest $D_{\text {eff }}$, while the NFW profile leads the most conservative value, and the variation is within $\sim 10 \%$. Therefore the difference in the DM density profile near the Galactic Center has little effect on the effective distance. If the CRs are approximated to be uniformly distributed in the $\mathrm{CR}$ propagation halo, the effective distance will be a constant as discussed in Eq. (11). These energy-independent effective distances for different DM density profiles are also shown in the right panel of Fig. 8. The values in this case are typically larger than that derived from spatial-dependent $\mathrm{CR}$ distributions, because the intensity of the inhomogeneous CRs decrease as the distance to the galactic plane increases.

\section{Appendix B: Parameters of the Primary CR Sources}

The primary source spectral parameters of the CR diffusion equation used in this work are listed in Tab. IV.

\section{Appendix C: Details of the Monte Carlo Simulation}

In this section we describe the details of the MC simulation in this work including the initial conditions, the trajectory simulation, and the reconstruction of the underground CRDM flux. 


\begin{tabular}{|c|c|c|c|c|c|c|}
\hline Nucleus & $\gamma_{0}^{R_{0}(\mathrm{GV})} s_{0}$ & $\gamma_{1}^{R_{1}(\mathrm{GV})} s_{1}$ & $\gamma_{2}^{R_{2}^{R_{2}(\mathrm{GV})} s_{2}}$ & $\gamma_{3}^{R_{3}(\mathrm{TV}}$ & ${ }^{\mathrm{V})} s_{3}$ & $\gamma_{4}$ \\
\hline${ }_{1} \mathrm{H}$ & $2.24^{0.95} 0.29$ & $1.70^{6.97} 0.22$ & $2.44^{400} 0.09$ & $2.19^{16}$ & 0.09 & 2.37 \\
\hline${ }_{2} \mathrm{He}$ & $2.05^{1.00} 0.26$ & $1.76^{7.49} 0.33$ & $2.41^{340} 0.13$ & $2.12^{30}$ & 0.10 & 2.37 \\
\hline${ }_{3}^{7} \mathrm{Li}$ & $\ldots$ & $1.10^{12.0} 0.16$ & $2.72^{355} 0.13$ & 1.90 & & \\
\hline${ }_{6} \mathrm{C}$ & $1.00^{1.10} 0.19$ & $1.98^{6.54} 0.31$ & $2.43^{348} 0.17$ & 2.12 & $\cdots$ & . \\
\hline${ }_{7}^{14} \mathrm{~N}$ & $1.00^{1.30} 0.17$ & $1.96^{7.00} 0.20$ & $2.46^{300} 0.17$ & 1.90 & $\ldots$ & $\ldots$ \\
\hline${ }_{8} \mathrm{O}$ & $0.95^{0.90} 0.18$ & $1.99^{7.50} 0.30$ & $2.46^{365} 0.17$ & 2.13 & $\ldots$ & $\ldots$ \\
\hline${ }_{9}^{19} \mathrm{~F}$ & $0.10^{1.00} 0.30$ & $1.00^{3.80} 0.32$ & $3.80^{8.0} 0.35$ & 3.12 & $\ldots$ & $\ldots$ \\
\hline${ }_{10} \mathrm{Ne}$ & $0.60^{1.15} 0.17$ & $1.92^{9.42} 0.26$ & $2.44^{355} 0.17$ & 1.97 & $\ldots$ & $\ldots$ \\
\hline${ }_{11} \mathrm{Na}$ & $0.50^{0.75} 0.17$ & $1.98^{7.00} 0.21$ & $2.49^{355} 0.17$ & 2.14 & $\cdots$ & $\ldots$ \\
\hline${ }_{12} \mathrm{Mg}$ & $0.20^{0.85} 0.12$ & $1.99^{7.00} 0.23$ & $2.48^{355} 0.17$ & 2.15 & $\cdots$ & $\ldots$ \\
\hline${ }_{13} \mathrm{Al}$ & $0.20^{0.60} 0.17$ & $2.04^{7.00} 0.20$ & $2.48^{355} 0.17$ & 2.14 & $\ldots$ & $\ldots$ \\
\hline${ }_{14} \mathrm{Si}$ & $0.20^{0.85} 0.17$ & $1.97^{7.00} 0.26$ & $2.47^{355} 0.17$ & 2.19 & $\ldots$ & $\ldots$ \\
\hline${ }_{15} \mathrm{P}$ & $0.25^{1.60} 0.19$ & $1.95^{7.00} 0.20$ & $2.48^{355} 0.17$ & 2.14 & $\ldots$ & $\ldots$ \\
\hline${ }_{16} \mathrm{~S}$ & $0.80^{1.30} 0.17$ & $1.96^{7.00} 0.20$ & $2.49^{355} 0.17$ & 2.14 & . . & $\ldots$ \\
\hline${ }_{17} \mathrm{Cl}$ & $1.10^{1.50} 0.17$ & $1.98^{7.20} 0.20$ & $2.53^{355} 0.17$ & 2.14 & & $\ldots$ \\
\hline${ }_{18} \mathrm{Ar}$ & $0.20^{1.30} 0.17$ & $1.96^{7.00} 0.20$ & $2.46^{355} 0.17$ & 2.09 & . & $\ldots$ \\
\hline${ }_{19} \mathrm{~K}$ & $0.20^{1.40} 0.15$ & $1.96^{7.00} 0.20$ & $2.53^{355} 0.17$ & 2.14 & & . \\
\hline${ }_{20} \mathrm{Ca}$ & $0.30^{1.00} 0.11$ & $2.07^{7.00} 0.20$ & $2.48^{355} 0.17$ & 2.14 & & $\ldots$ \\
\hline${ }_{21} \mathrm{Sc}$ & $0.20^{1.40} 0.17$ & $1.97^{7.00} 0.22$ & $2.53^{355} 0.17$ & 2.14 & $\ldots$ & $\ldots$ \\
\hline${ }_{22} \mathrm{Ti}$ & $1.50^{0.90} 0.17$ & $1.98^{7.00} 0.22$ & $2.57^{355} 0.17$ & 2.14 & & $\ldots$ \\
\hline${ }_{23} \mathrm{~V}$ & $1.10^{0.80} 0.17$ & $1.98^{7.00} 0.22$ & $2.53^{355} 0.17$ & 2.14 & & $\ldots$ \\
\hline${ }_{24} \mathrm{Cr}$ & $1.70^{0.65} 0.17$ & $1.99^{7.00} 0.20$ & $2.48^{355} 0.17$ & 2.14 & & $\ldots$ \\
\hline${ }_{25} \mathrm{Mn}$ & $0.20^{0.85} 0.10$ & $2.08^{7.00} 0.20$ & $2.48^{355} 0.17$ & 2.14 & & $\ldots$ \\
\hline${ }_{26} \mathrm{Fe}$ & $0.95^{2.00} 0.20$ & $3.62^{2.94} 0.10$ & $2.05^{17.0} 0.18$ & $2.452^{c}$ & 0.3550 .17 & 2.23 \\
\hline${ }_{27} \mathrm{Co}$ & $0.80^{0.70} 0.15$ & $1.98^{7.00} 0.20$ & $2.49^{355} 0.17$ & 2.14 & & \\
\hline${ }_{28} \mathrm{Ni}$ & $1.50^{0.65} 0.17$ & $1.98^{7.00} 0.20$ & $2.48^{355} 0.17$ & 2.14 & & $\ldots$ \\
\hline
\end{tabular}

TAB. IV: The primary source spectral parameters of the CR diffusion equation taken from Ref. [40] with updated parameters of Fe from Ref. [51] and F from Ref. [52].

\section{Initial conditions}

In this work, we only consider the angular-averaged CRDM flux as most of the current DM direct detection experiments cannot distinguish the arrival direction of DM. The initial momenta of the particles are set to be isotropic with the kinetic energy sampled from the normalized surface flux in Eq. (5). The isotropy of the injection is guaranteed by appropriately choosing the initial position [73]. Once a random direction is sampled isotropically, the initial position is chosen randomly on a circular disk of radius $R_{\oplus}$ at a distance $R$ from the Earth center and perpendicular to that direction. Here $R_{\oplus} \approx 6371 \mathrm{~km}$ is the radius of the Earth, and $R=1.1 R_{\oplus}$ is an arbitrary distance greater than $R_{\oplus}$. The momentum is then taken to be along that direction and point to the Earth. We have checked that this 
approach ensures that the same isotropic flux is obtained inside the Earth in the case where the Earth is transparent to DM particles.

\section{Trajectory simulation}

The simulation of the trajectory of DM particles inside the Earth is composed of two processes, free-propagation and scattering. The free-propagations are considered as straight lines of length $\hat{l}$ between two consecutive collisions since gravity can be safely neglected. The cumulative distribution function $(\mathrm{CDF})$ of the random variable $\hat{l}$ is

$$
F(L)=P(\hat{l}<L)=1-\exp \left(-\int_{0}^{L} \frac{d x}{\ell}\right),
$$

where the mean free path $\ell$ is defined as

$$
\ell^{-1}=\sum_{N} \ell_{N}^{-1} \equiv \sum_{N} n_{N} \sigma_{\chi N}
$$

where $n_{N}$ is the number density of nuclear species $N$, and $\sigma_{\chi N}$ is the total cross section defined in Eq. (25). We draw samples of the free path by using the inverse CDF method

$$
L=F^{-1}(\xi),
$$

where $\xi$ is a random number drawn from a uniform distribution between 0 and 1 . Explicitly, we solve $L$ from the equation

$$
\int_{0}^{L} \frac{d x}{\ell}=-\ln (1-\xi) .
$$

The specific form of $\ell$ depends on the cross section and the Earth model. For the homogeneous Earth model considered in this work, $\ell$ is independent of position, and the free path is solved as

$$
L=-\ell \ln (1-\xi) .
$$

After traveling freely for a distance of $L$, the DM particle scatters with the nuclei in the Earth. Which kind of nucleus to collide with is determined by the collision probability

$$
P_{N}=\frac{n_{N} \sigma_{\chi N}}{\sum_{N^{\prime}} n_{N^{\prime}} \sigma_{\chi N^{\prime}}}=\frac{\ell}{\ell_{N}}
$$

Then we simulate the scattering between DM and the nucleus $N$ according to the differential cross section. The probability density function (PDF) of the momentum transfer squared $q^{2}$ is

$$
p\left(q^{2}\right)=\frac{1}{\sigma_{\chi N}} \frac{d \sigma_{\chi N}}{d q^{2}}
$$

For the cross section considered in this work in Eq. (17), sampling via inversion of the CDF simplifies to solve $q^{2}$ from the equation

$$
\frac{\int_{0}^{q^{2}} F_{N}^{2}\left(q^{\prime 2}\right) d q^{\prime 2}}{\int_{0}^{q_{\max }^{2}} F_{N}^{2}\left(q^{\prime 2}\right) d q^{\prime 2}}=\xi
$$


where $q_{\max }^{2}=2 m_{N} T_{N}^{\max }$ with $T_{N}^{\max }$ from Eq. (1). After the recoil energy $T_{N}=q^{2} / 2 m_{N}$ was sampled, the scattering angle of DM particle in the laboratory frame can be calculated from kinematics as

$$
\cos \theta=\frac{p_{i}^{2}-T_{N}\left(T_{i}+m_{\chi}+m_{N}\right)}{p_{i} p_{f}}
$$

where $p_{i(f)}=\sqrt{T_{i(f)}^{2}+2 m_{\chi} T_{i(f)}}$ is the initial (final) momentum of DM with $T_{f}=T_{i}-T_{N}$. The direction of the final momentum is determined by supplementing a uniformly random azimuthal angle from 0 to $2 \pi$.

\section{Underground flux reconstruction}

With the continuous injection of DM particles, we expect them to form a stable distribution inside the earth. But simulating the time evolution is time-consuming because the simulated trajectories before reaching equilibrium are all discarded. On the other hand, all the trajectories can be regarded as an ensemble to reconstruct the underground flux. We record the crossing events of particles passing through a given surface $\Delta S$ underground to calculate the corresponding flux. An effective time interval $\Delta t$ can be determined by the injected particle number and the flux. Assuming time translation invariance, the collected events in the simulation are equivalent to those in the time interval $\Delta t$ from a continuous injection scenario. Then the underground flux is related to these crossing events as

$$
\frac{d \Phi_{\chi}^{d}}{d T_{\chi}}\left|\cos \theta_{i}\right|=\frac{\Delta N_{i}}{\Delta T_{\chi} \Delta S \Delta \Omega \Delta t},
$$

where $\Delta N_{i}$ is the number of events crossing the area $\Delta S$ with energy in the $i$ th energy bin $\left(T_{i}, T_{i}+\Delta T_{\chi}\right)$ and from a solid angle $\Delta \Omega$ of the arrival direction. $\theta_{i}$ is the angle of the arrival direction.

We sample $N_{\text {sim }}$ DM particles using the method described in Appendix C 1 with energies in an interval $\left(T_{a}, T_{b}\right)$. We trace each particle during the simulation and record the crossing events whenever it passes through an underground spherical surface at a depth of $d$ centered at the center of the Earth. Each crossing event contains the kinetic energy, position, and momentum direction of the particle. In this case, we have $\Delta \Omega=4 \pi$ and $\Delta S=S_{d}=$ $4 \pi\left(R_{\oplus}-d\right)^{2}$, which is the area of the underground sphere. The effective injection time $\Delta t$ is determined as

$$
\Delta t=\frac{N_{\text {sim }}}{\pi S_{0} \Phi_{0}^{\text {norm }}},
$$

where $\Phi_{0}^{\text {norm }}=\int_{T_{a}}^{T_{b}} \frac{d \Phi_{\chi}^{0}}{d T_{\chi}} d T_{\chi}$ is the integral flux of CRDM at the surface of the Earth, and $S_{0}=4 \pi R_{\oplus}^{2}$ is the Earth's surface area. Note that this expression is derived using the assumption that the flux is isotropic and we only inject particles from outside the Earth. Combining these terms we can write the angular-averaged underground flux as

$$
\frac{d \bar{\Phi}_{\chi}^{d}}{d T_{\chi}}=\frac{S_{0} \Phi_{0}^{\mathrm{norm}}}{4 S_{d} N_{\mathrm{sim}}} \sum_{j} w_{j} f(T),
$$


where $w_{j}=1 /\left|\cos \theta_{j}\right|$ is the weight of event $j$, and $f(T)$ is the normalized probability density function of kinetic energy constructed from the sample points weighted by $w_{j}$. If importance sampling (IS) is implemented in the simulation, the corresponding weights need to be transferred to $w_{j}$. We adopted IS to improve the sampling efficiency of the high-energy part of the injection flux, which is summarized in Appendix E.

Note that the formula of Eq. (C12) is valid under the condition that the initial energy of the particles is sampled from the flux, which is essentially different from sampling from velocity distribution. But if we regard sampling from the velocity distribution as an IS from the flux, the above analysis can be followed by acquiring a weight for each DM particle. The weight is proportional to the initial speed $v$ since the flux $\Phi \propto v f(\boldsymbol{v})$. The DaMaSCUS code samples initial state from the velocity distribution with this weight missed in the original version [73], which has been corrected in the latest version [89]. Our flux-based method is consistent with the updated version.

\section{Appendix D: Comparison with the DaMaSCUS-CRUST Code for Halo DM}

In this section we give the results of the Earth attenuation of the non-relativistic halo DM and compare them with the results from the DaMaSCUS-CRUST [68]. We show that the results are in good agreement. We further show that the attenuation in the MC simulation can be stronger than that in the analytic method for light halo DM. Our code is publicly available at the DarkProp webpage [34].

The simulation procedure is essentially the same as that for CRDM described in Appendix $\mathrm{C}$, except that the initial condition is based on the Maxwellian velocity distribution of the standard halo model (SHM). The most probable speed and the escape velocity are taken to be $v_{0}=220 \mathrm{kms}$ and $v_{\mathrm{esc}}=544 \mathrm{~km} / \mathrm{s}$ respectively. The initial velocity is sampled from the isotropic SHM velocity distribution and then subtracted by a fixed oriented Earth velocity with a magnitude of $240 \mathrm{~km} / \mathrm{s}$. We perform two sets of simulations for DM particle mass $m_{\chi}=10$ and $0.5 \mathrm{GeV}$. Each set of the simulation involve a number of cross sections. The underground DM fluxes at a depth of $1.4 \mathrm{~km}$ are reconstructed. We calculate the recoil events number of the DM-xenon nucleus scatterings in the recoil energy range of $0.5-40(0.013-40) \mathrm{keV}$ for DM particle mass $m_{\chi}=10(0.5) \mathrm{GeV}$ with a exposure of 35.6 ton - days. The recoil energy ranges are chosen to be different and adjusted for producing the same events numbers in two DM particle mass cases when Earth attenuation is absent. The threshold velocity for termination of the simulation is set to be $566(536) \mathrm{km} / \mathrm{s}$ for $m_{\chi}=10(0.5) \mathrm{GeV}$. The nuclear form factor is neglected for these light non-relativistic DMs.

The recoil events numbers derived from the MC method as a function of cross section are shown in Fig. 9, which are compared with the results using the analytic method. The unattenuated events number on the surface of the Earth is also shown for comparison. In the case of $m_{\chi}=10 \mathrm{GeV}$, the number of events drops rapidly when the cross section is sufficiently large and drops later in the simulation than that in the analytic approximation which is in good agreement with DaMaSCUS-CRUST [68]. On the other hand, for lighter DM with mass $m_{\chi}=0.5 \mathrm{GeV}$, the number of events in the simulation drops before that in 


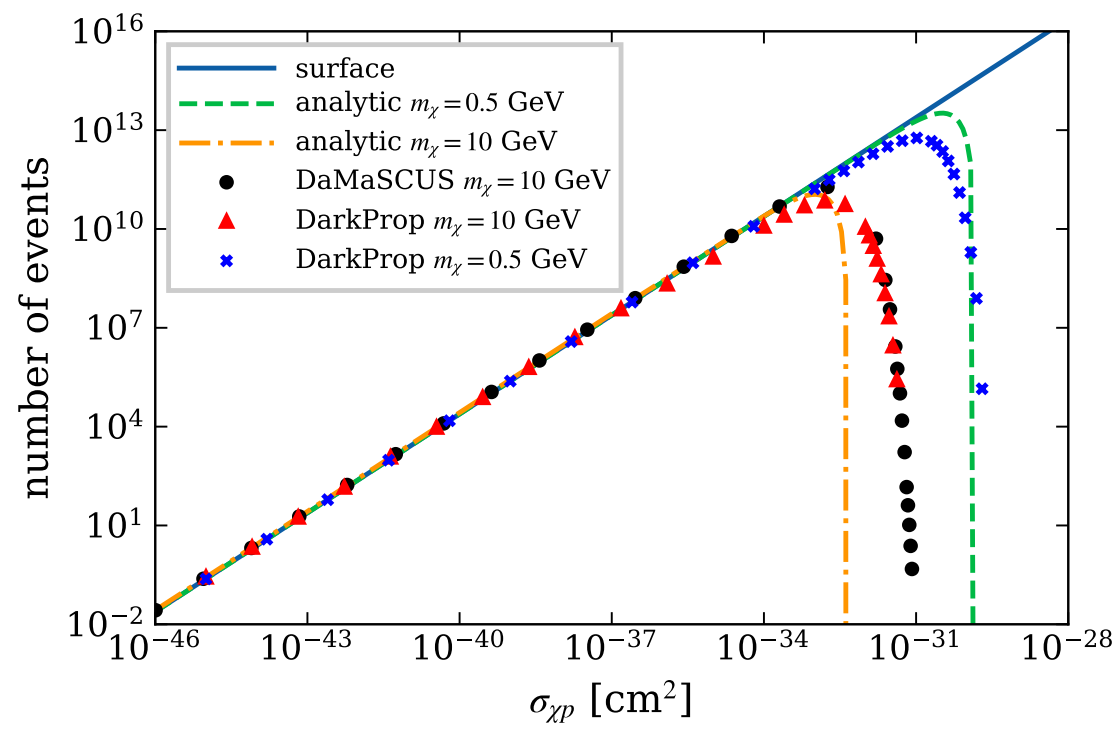

FIG. 9: Recoil events number predicted for a xenon based DM detector as a function of cross section for halo DM with masses $m_{\chi}=10$ and $0.5 \mathrm{GeV}$. The exposure is assumed to be 35.6 ton - days, and the considered nuclear recoil energy range is $[5,40] \mathrm{keV}$ for $m_{\chi}=10 \mathrm{GeV}$ and $[0.013,40] \mathrm{keV}$ for $m_{\chi}=0.5 \mathrm{GeV}$ respectively. The green dashed (orange dash-dotted) line represents the events number calculated using analytic method for $m_{\chi}=10(0.5) \mathrm{GeV}$. The events numbers at the surface for the two DM particle masses are the same due to the specific recoil energy ranges chosen and shown with a blue solid line. The result using MC simulation for $m_{\chi}=10(0.5) \mathrm{GeV}$ is shown as red triangles (blue crosses). The result from DaMaSCUS-CRUST [68] is shown as black circles for comparison.

the analytic approximation as the cross section increases, which is opposite to the case of $m_{\chi}=10 \mathrm{GeV}$. This result shows that the attenuation in the MC simulation can be stronger than that in the analytic method for light halo DM due to larger scattering angles in the laboratory frame.

\section{Appendix E: Importance Sampling}

The CRDM flux is approximately a power law spectrum with a power index of about -2 [24], therefore dropping quickly with energy increases. If we sample energy points directly from the normalized flux in a wide energy range, the energies at the high-energy end will be difficult to obtain due to the extremely low probability. We use importance sampling method to overcome this problem since we are concerned about the Earth attenuation of high-energy CRDM.

Suppose $f(T)$ is the probability distribution of energy we need to sample. We do not sample directly from this distribution but from an artificial flatter distribution $g(T)$, in which the relative probability of the high-energy part is higher. Then we assign a weight $w_{i}=f\left(T_{i}\right) / g\left(T_{i}\right)$ to each sample $T_{i}$. The original distribution $f(T)$ should be reconstructable 
using these weighted samples. In MC simulation, these weights need to transfer to the final sample analysis.

In our problem, the initial energy of each trajectory is sampled from the surface CRDM flux. We choose $g(T)$ as a logarithmic uniform distribution on the region-of-interest $\left(T_{a}, T_{b}\right)$

$$
g(T)=\frac{1}{\left(\ln T_{b}-\ln T_{a}\right) T}
$$

Then the energy points $T_{j}$ are obtained directly by the exponential of samples from a uniform distribution on $\left(\ln T_{a}, \ln T_{b}\right)$ with the weights

$$
w_{j}^{\mathrm{IS}}=\frac{T_{j}}{\Phi_{0}^{\text {norm }}} \frac{d \Phi_{\chi}^{0}}{d T_{j}} \ln \left(\frac{T_{b}}{T_{a}}\right) .
$$

Correspondingly, the weights in Eq. (C12) for calculating the underground flux need to be modified to

$$
w_{j}=\frac{w_{j}^{\mathrm{IS}}}{\left|\cos \theta_{j}\right|} .
$$

If a particle crosses the underground surface multiple times, these events will acquire the same weight $w^{\mathrm{IS}}$ according to the initial energy.

[1] XENON Collaboration, E. Aprile et al., "Dark Matter Search Results from a One Ton-Year Exposure of XENON1T," Phys. Rev. Lett. 121 no. 11, (2018) 111302, arXiv:1805.12562 [astro-ph.CO].

[2] PandaX-II Collaboration, A. Tan et al., "Dark Matter Results from First 98.7 Days of Data from the PandaX-II Experiment," Phys. Rev. Lett. 117 no. 12, (2016) 121303, arXiv:1607.07400 [hep-ex].

[3] C. Kouvaris and J. Pradler, "Probing sub-GeV Dark Matter with conventional detectors," Phys. Rev. Lett. 118 no. 3, (2017) 031803, arXiv:1607.01789 [hep-ph].

[4] M. Ibe, W. Nakano, Y. Shoji, and K. Suzuki, "Migdal Effect in Dark Matter Direct Detection Experiments," JHEP 03 (2018) 194, arXiv: 1707.07258 [hep-ph].

[5] M. J. Dolan, F. Kahlhoefer, and C. McCabe, "Directly detecting sub-GeV dark matter with electrons from nuclear scattering," Phys. Rev. Lett. 121 no. 10, (2018) 101801, arXiv:1711.09906 [hep-ph].

[6] V. Gluscevic and K. K. Boddy, "Constraints on Scattering of keV-TeV Dark Matter with Protons in the Early Universe," Phys. Rev. Lett. 121 no. 8, (2018) 081301, arXiv:1712.07133 [astro-ph.CO].

[7] D. Wadekar and G. R. Farrar, "First astrophysical constraints on dark matter interactions with ordinary matter at low relative velocity," arXiv:1903.12190 [hep-ph].

[8] A. Bhoonah, J. Bramante, F. Elahi, and S. Schon, "Calorimetric Dark Matter Detection With Galactic Center Gas Clouds," Phys. Rev. Lett. 121 no. 13, (2018) 131101, arXiv:1806.06857 [hep-ph]. 
[9] E. O. Nadler, V. Gluscevic, K. K. Boddy, and R. H. Wechsler, "Constraints on Dark Matter Microphysics from the Milky Way Satellite Population," Astrophys. J. Lett. 878 no. 2, (2019) 32, arXiv:1904.10000 [astro-ph.C0]. [Erratum: Astrophys.J.Lett. 897, L46 (2020), Erratum: Astrophys.J. 897, L46 (2020)].

[10] R. K. Leane and J. Smirnov, "Exoplanets as Sub-GeV Dark Matter Detectors," Phys. Rev. Lett. 126 no. 16, (2021) 161101, arXiv:2010.00015 [hep-ph].

[11] C. V. Cappiello, K. C. Ng, and J. F. Beacom, "Reverse Direct Detection: Cosmic Ray Scattering With Light Dark Matter," Phys. Rev. D 99 no. 6, (2019) 063004, arXiv:1810.07705 [hep-ph].

[12] R. H. Cyburt, B. D. Fields, V. Pavlidou, and B. D. Wandelt, "Constraining strong baryon dark matter interactions with primordial nucleosynthesis and cosmic rays," Phys. Rev. D 65 (2002) 123503, arXiv:astro-ph/0203240.

[13] D. Hooper and S. D. McDermott, "Robust Constraints and Novel Gamma-Ray Signatures of Dark Matter That Interacts Strongly With Nucleons," Phys. Rev. D 97 no. 11, (2018) 115006, arXiv: 1802.03025 [hep-ph].

[14] T. Bringmann and M. Pospelov, "Novel direct detection constraints on light dark matter," Phys. Rev. Lett. 122 no. 17, (2019) 171801, arXiv:1810.10543 [hep-ph].

[15] Y. Ema, F. Sala, and R. Sato, "Light Dark Matter at Neutrino Experiments," Phys. Rev. Lett. 122 no. 18, (2019) 181802, arXiv:1811.00520 [hep-ph].

[16] C. Cappiello and J. F. Beacom, "Strong New Limits on Light Dark Matter from Neutrino Experiments," Phys. Rev. D 100 no. 10, (2019) 103011, arXiv:1906.11283 [hep-ph].

[17] W. Wang, L. Wu, J. M. Yang, H. Zhou, and B. Zhu, "Cosmic ray boosted sub-GeV gravitationally interacting dark matter in direct detection," JHEP 12 (2020) 072, arXiv:1912.09904 [hep-ph]. [Erratum: JHEP 02, 052 (2021)].

[18] PROSPECT, (PROSPECT Collaboration)* Collaboration, M. Andriamirado et al., "Limits on sub-GeV dark matter from the PROSPECT reactor antineutrino experiment," Phys. Rev. D 104 no. 1, (2021) 012009, arXiv:2104.11219 [hep-ex].

[19] S.-F. Ge, J.-L. Liu, Q. Yuan, and N. Zhou, "Boosted Diurnal Effect of Sub-GeV Dark Matter at Direct Detection Experiment," arXiv:2005.09480 [hep-ph].

[20] J. B. Dent, B. Dutta, J. L. Newstead, and I. M. Shoemaker, "Bounds on Cosmic Ray-Boosted Dark Matter in Simplified Models and its Corresponding Neutrino-Floor," Phys. Rev. D 101 no. 11, (2020) 116007, arXiv:1907.03782 [hep-ph].

[21] K. Bondarenko, A. Boyarsky, T. Bringmann, M. Hufnagel, K. Schmidt-Hoberg, and A. Sokolenko, "Direct detection and complementary constraints for sub-GeV dark matter," JHEP 03 (2020) 118, arXiv:1909.08632 [hep-ph].

[22] G. Guo, Y.-L. S. Tsai, M.-R. Wu, and Q. Yuan, "Elastic and Inelastic Scattering of Cosmic-Rays on Sub-GeV Dark Matter," Phys. Rev. D 102 no. 10, (2020) 103004, arXiv:2008.12137 [astro-ph.HE].

[23] G. Guo, Y.-L. S. Tsai, and M.-R. Wu, "Probing cosmic-ray accelerated light dark matter with IceCube," JCAP 10 (2020) 049, arXiv:2004.03161 [astro-ph.HE].

[24] C. Xia, Y.-H. Xu, and Y.-F. Zhou, "Constraining light dark matter upscattered by ultrahigh-energy cosmic rays," Nucl. Phys. B 969 (2021) 115470, arXiv: 2009.00353 
[hep-ph].

[25] J. Alvey, M. Campos, M. Fairbairn, and T. You, "Detecting Light Dark Matter via Inelastic Cosmic Ray Collisions," Phys. Rev. Lett. 123 (2019) 261802, arXiv:1905.05776 [hep-ph].

[26] L. Su, W. Wang, L. Wu, J. M. Yang, and B. Zhu, "Atmospheric Dark Matter and Xenon1T Excess," Phys. Rev. D 102 no. 11, (2020) 115028, arXiv:2006.11837 [hep-ph].

[27] Y. Ema, F. Sala, and R. Sato, "Neutrino experiments probe hadrophilic light dark matter," SciPost Phys. 10 no. 3, (2021) 072, arXiv:2011.01939 [hep-ph].

[28] N. F. Bell, J. B. Dent, B. Dutta, S. Ghosh, J. Kumar, J. L. Newstead, and I. M. Shoemaker, "Cosmic-ray upscattered inelastic dark matter," Phys. Rev. D 104 (2021) 076020, arXiv:2108.00583 [hep-ph].

[29] G. Krnjaic and S. D. McDermott, "Implications of BBN Bounds for Cosmic Ray Upscattered Dark Matter," Phys. Rev. D 101 no. 12, (2020) 123022, arXiv:1908.00007 [hep-ph].

[30] T. R. Slatyer and C.-L. Wu, "Early-Universe constraints on dark matter-baryon scattering and their implications for a global $21 \mathrm{~cm}$ signal," Phys. Rev. D 98 no. 2, (2018) 023013, arXiv:1803.09734 [astro-ph.CO].

[31] K. K. Boddy and V. Gluscevic, "First Cosmological Constraint on the Effective Theory of Dark Matter-Proton Interactions," Phys. Rev. D 98 no. 8, (2018) 083510, arXiv:1801.08609 [astro-ph.CO].

[32] W. L. Xu, C. Dvorkin, and A. Chael, "Probing sub-GeV Dark Matter-Baryon Scattering with Cosmological Observables," Phys. Rev. D 97 no. 10, (2018) 103530, arXiv:1802.06788 [astro-ph.CO].

[33] R. Murgia, V. Iršič, and M. Viel, "Novel constraints on noncold, nonthermal dark matter from Lyman- $\alpha$ forest data," Phys. Rev. D 98 no. 8, (2018) 083540, arXiv:1806.08371 [astro-ph.CO].

[34] C. Xia, "DarkProp-v0.1." http://yfzhou.itp.ac.cn/darkprop, 2021.

[35] V. S. Berezinsky, S. V. Bulanov, V. A. Dogiel, and V. S. Ptuskin, Astrophysics of cosmic rays. 1990.

[36] A. W. Strong, I. V. Moskalenko, and V. S. Ptuskin, "Cosmic-ray propagation and interactions in the Galaxy," Ann. Rev. Nucl. Part. Sci. 57 (2007) 285-327, arXiv: astro-ph/0701517.

[37] A. Strong and I. Moskalenko, "Propagation of cosmic-ray nucleons in the galaxy," Astrophys. J. 509 (1998) 212-228, arXiv:astro-ph/9807150.

[38] I. V. Moskalenko and A. W. Strong, "Production and propagation of cosmic ray positrons and electrons," Astrophys. J. 493 (1998) 694-707, arXiv: astro-ph/9710124.

[39] M. J. Boschini et al., "Solution of heliospheric propagation: unveiling the local interstellar spectra of cosmic ray species," Astrophys. J. 840 no. 2, (2017) 115, arXiv:1704.06337 [astro-ph.HE].

[40] M. Boschini et al., "Inference of the Local Interstellar Spectra of Cosmic Ray Nuclei $\mathrm{Z}<=28$ with the GalProp-HelMod Framework," Astrophys. J. Suppl. 250 no. 2, (2020) 27, arXiv:2006.01337 [astro-ph.HE].

[41] A. C. Cummings, E. C. Stone, B. C. Heikkila, N. Lal, W. R. Webber, G. Jóhannesson, I. V. Moskalenko, E. Orlando, and T. A. Porter, "Galactic Cosmic Rays in the Local Interstellar 
Medium: Voyager 1 Observations and Model Results," Astrophys. J. 831 no. 1, (2016) 18.

[42] M. E. Wiedenbeck, N. E. Yanasak, A. C. Cummings, A. J. Davis, J. S. George, R. A. Leske, R. A. Mewaldt, E. C. Stone, P. L. Hink, M. H. Israel, M. Lijowski, E. R. Christian, and T. T. Von Rosenvinge, "The origin of primary cosmic rays: Constraints from ace elemental and isotopic composition observations," in The Astrophysics of Galactic Cosmic Rays, R. Diehl, E. Parizot, R. Kallenbach, and R. Von Steiger, eds., pp. 15-26. Springer Netherlands, Dordrecht, 2001.

[43] H. S. Ahn et al., "Measurements of cosmic-ray secondary nuclei at high energies with the first flight of the CREAM balloon-borne experiment," Astropart. Phys. 30 (2008) 133-141, arXiv:0808.1718 [astro-ph].

[44] H. S. Ahn et al., "Energy spectra of cosmic-ray nuclei at high energies," Astrophys. J. 707 (2009) 593-603, arXiv:0911.1889 [astro-ph.HE].

[45] N. Gorbunov et al., "Energy spectra of abundant cosmic-ray nuclei in the NUCLEON experiment," Adv. Space Res. 64 no. 12, (2019) 2546-2558, arXiv:1809.05333 [astro-ph. IM].

[46] V. Grebenyuk et al., "Secondary cosmic rays in the NUCLEON space experiment," Adv. Space Res. 64 (2019) 2559-2563, arXiv:1809.09665 [astro-ph.HE].

[47] CALET Collaboration, O. Adriani et al., "Direct Measurement of the Cosmic-Ray Proton Spectrum from $50 \mathrm{GeV}$ to $10 \mathrm{TeV}$ with the Calorimetric Electron Telescope on the International Space Station," Phys. Rev. Lett. 122 no. 18, (2019) 181102, arXiv:1905.04229 [astro-ph.HE].

[48] J. J. Engelmann, P. Ferrando, A. Soutoul, P. Goret, and E. Juliusson, "Charge composition and energy spectra of cosmic-ray for elements from Be to NI - Results from HEAO-3-C2," Astron. Astrophys. 233 (1990) 96-111.

[49] DAMPE Collaboration, Q. An et al., "Measurement of the cosmic-ray proton spectrum from $40 \mathrm{GeV}$ to $100 \mathrm{TeV}$ with the DAMPE satellite," Sci. Adv. 5 no. 9, (2019) eaax3793, arXiv:1909.12860 [astro-ph.HE].

[50] AMS Collaboration, M. Aguilar et al., "Observation of New Properties of Secondary Cosmic Rays Lithium, Beryllium, and Boron by the Alpha Magnetic Spectrometer on the International Space Station," Phys. Rev. Lett. 120 no. 2, (2018) 021101.

[51] M. J. Boschini et al., "The Discovery of a Low-energy Excess in Cosmic-Ray Iron: Evidence of the Past Supernova Activity in the Local Bubble," Astrophys. J. 913 no. 1, (2021) 5, arXiv:2101.12735 [astro-ph.HE].

[52] M. J. Boschini et al., "A Hint of a Low-Energy Excess in Cosmic-Ray Fluorine," arXiv:2106.01626 [astro-ph.HE].

[53] AMS Collaboration, M. Aguilar et al., "Properties of Iron Primary Cosmic Rays: Results from the Alpha Magnetic Spectrometer," Phys. Rev. Lett. 126 no. 4, (2021) 041104.

[54] AMS Collaboration, M. Aguilar et al., "Properties of Heavy Secondary Fluorine Cosmic Rays: Results from the Alpha Magnetic Spectrometer," Phys. Rev. Lett. 126 no. 8, (2021) 081102.

[55] D. R. Lorimer et al., "The Parkes multibeam pulsar survey: VI. Discovery and timing of 142 pulsars and a Galactic population analysis," Mon. Not. Roy. Astron. Soc. 372 (2006) 
777-800, arXiv:astro-ph/0607640.

[56] AMS Collaboration, M. Aguilar et al., "Precision Measurement of the Proton Flux in Primary Cosmic Rays from Rigidity 1 GV to 1.8 TV with the Alpha Magnetic Spectrometer on the International Space Station," Phys. Rev. Lett. 114 (2015) 171103.

[57] AMS Collaboration, M. Aguilar et al., "Precision Measurement of the Helium Flux in Primary Cosmic Rays of Rigidities $1.9 \mathrm{GV}$ to $3 \mathrm{TV}$ with the Alpha Magnetic Spectrometer on the International Space Station," Phys. Rev. Lett. 115 no. 21, (2015) 211101.

[58] AMS Collaboration, M. Aguilar et al., "Observation of the Identical Rigidity Dependence of $\mathrm{He}, \mathrm{C}$, and O Cosmic Rays at High Rigidities by the Alpha Magnetic Spectrometer on the International Space Station," Phys. Rev. Lett. 119 no. 25, (2017) 251101.

[59] J. F. Navarro, C. S. Frenk, and S. D. White, "The Structure of cold dark matter halos," Astrophys. J. 462 (1996) 563-575, arXiv:astro-ph/9508025.

[60] Fermi-LAT Collaboration, M. Ackermann et al., "Constraints on the Galactic Halo Dark Matter from Fermi-LAT Diffuse Measurements," Astrophys. J. 761 (2012) 91, arXiv:1205.6474 [astro-ph.CO].

[61] G. Jungman, M. Kamionkowski, and K. Griest, "Supersymmetric dark matter," Phys. Rept. 267 (1996) 195-373, arXiv:hep-ph/9506380.

[62] C. Perdrisat, V. Punjabi, and M. Vanderhaeghen, "Nucleon Electromagnetic Form Factors," Prog. Part. Nucl. Phys. 59 (2007) 694-764, arXiv:hep-ph/0612014.

[63] R. H. Helm, "Inelastic and Elastic Scattering of 187-Mev Electrons from Selected Even-Even Nuclei," Phys. Rev. 104 (1956) 1466-1475.

[64] J. Lewin and P. Smith, "Review of mathematics, numerical factors, and corrections for dark matter experiments based on elastic nuclear recoil," Astropart. Phys. 6 (1996) 87-112.

[65] PandaX-II Collaboration, X. Cui et al., "A Search for the Cosmic Ray Boosted Sub-GeV Dark Matter at the PandaX-II Experiment," arXiv:2112.08957 [hep-ex].

[66] G. D. Starkman, A. Gould, R. Esmailzadeh, and S. Dimopoulos, "Opening the Window on Strongly Interacting Dark Matter," Phys. Rev. D 41 (1990) 3594.

[67] C. Kouvaris and I. M. Shoemaker, "Daily modulation as a smoking gun of dark matter with significant stopping rate," Phys. Rev. D 90 (2014) 095011, arXiv:1405.1729 [hep-ph].

[68] T. Emken and C. Kouvaris, "How blind are underground and surface detectors to strongly interacting Dark Matter?," Phys. Rev. D 97 no. 11, (2018) 115047, arXiv:1802.04764 [hep-ph].

[69] B. J. Kavanagh, R. Catena, and C. Kouvaris, "Signatures of Earth-scattering in the direct detection of Dark Matter," JCAP 01 (2017) 012, arXiv:1611.05453 [hep-ph].

[70] B. J. Kavanagh, "Earth scattering of superheavy dark matter: Updated constraints from detectors old and new," Phys. Rev. D 97 no. 12, (2018) 123013, arXiv:1712.04901 [hep-ph].

[71] T. Bringmann, J. Edsjö, P. Gondolo, P. Ullio, and L. Bergström, "DarkSUSY 6 : An Advanced Tool to Compute Dark Matter Properties Numerically," JCAP 07 (2018) 033, arXiv: 1802.03399 [hep-ph].

[72] M. S. Mahdawi and G. R. Farrar, "Closing the window on $\sim \mathrm{GeV}$ Dark Matter with moderate ( $\mu$ b) interaction with nucleons," JCAP 12 (2017) 004, arXiv:1709.00430 [hep-ph]. 
[73] T. Emken and C. Kouvaris, "DaMaSCUS: The Impact of Underground Scatterings on Direct Detection of Light Dark Matter," JCAP 10 (2017) 031, arXiv:1706.02249 [hep-ph].

[74] A. M. Dziewonski and D. L. Anderson, "Preliminary reference earth model," Phys. Earth Planet. Interiors 25 (1981) 297-356.

[75] Y. Chen, B. Fornal, P. Sandick, J. Shu, X. Xue, Y. Zhao, and J. Zong, "Earth Shielding and Daily Modulation from Electrophilic Boosted Dark Matter," arXiv:2110.09685 [hep-ph].

[76] XENON Collaboration, E. Aprile et al., "Dark Matter Search Results from a One Ton-Year Exposure of XENON1T," Phys. Rev. Lett. 121 no. 11, (2018) 111302, arXiv:1805.12562 [astro-ph.CO].

[77] XENON Collaboration, E. Aprile et al., "XENON1T dark matter data analysis: Signal and background models and statistical inference," Phys. Rev. D 99 no. 11, (2019) 112009, arXiv:1902.11297 [physics.ins-det].

[78] XENON Collaboration, E. Aprile et al., "Light Dark Matter Search with Ionization Signals in XENON1T," Phys. Rev. Lett. 123 no. 25, (2019) 251801, arXiv:1907.11485 [hep-ex].

[79] A. M. Green, "Calculating exclusion limits for weakly interacting massive particle direct detection experiments without background subtraction," Phys. Rev. D 65 (2002) 023520, arXiv:astro-ph/0106555.

[80] C. Savage, G. Gelmini, P. Gondolo, and K. Freese, "Compatibility of DAMA/LIBRA dark matter detection with other searches," JCAP 04 (2009) 010, arXiv:0808.3607 [astro-ph].

[81] CRESST Collaboration, G. Angloher et al., "Results on light dark matter particles with a low-threshold CRESST-II detector," Eur. Phys. J. C 76 no. 1, (2016) 25, arXiv:1509.01515 [astro-ph.CO].

[82] CRESST Collaboration, G. Angloher et al., "Results on MeV-scale dark matter from a gram-scale cryogenic calorimeter operated above ground," Eur. Phys. J. C 77 no. 9, (2017) 637, arXiv:1707.06749 [astro-ph.CO].

[83] XENON Collaboration, E. Aprile et al., "First Dark Matter Search Results from the XENON1T Experiment," Phys. Rev. Lett. 119 no. 18, (2017) 181301, arXiv:1705.06655 [astro-ph.CO].

[84] XENON Collaboration, E. Aprile et al., "Search for Light Dark Matter Interactions Enhanced by the Migdal Effect or Bremsstrahlung in XENON1T," Phys. Rev. Lett. 123 no. 24, (2019) 241803, arXiv:1907.12771 [hep-ex].

[85] M. S. Mahdawi and G. R. Farrar, "Constraints on Dark Matter with a moderately large and velocity-dependent DM-nucleon cross-section," JCAP 10 (2018) 007, arXiv:1804.03073 [hep-ph].

[86] DES Collaboration, E. O. Nadler et al., "Milky Way Satellite Census. III. Constraints on Dark Matter Properties from Observations of Milky Way Satellite Galaxies," Phys. Rev. Lett. 126 (2021) 091101, arXiv:2008.00022 [astro-ph.CO].

[87] J. Einasto, "Dark Matter," 1, 2009. arXiv:0901.0632 [astro-ph.C0].

[88] J. N. Bahcall and R. M. Soneira, "The Universe at faint magnetidues. 2. Models for the predicted star counts," Astrophys. J. Suppl. 44 (1980) 73-110.

[89] https://github.com/temken/DaMaSCUS 